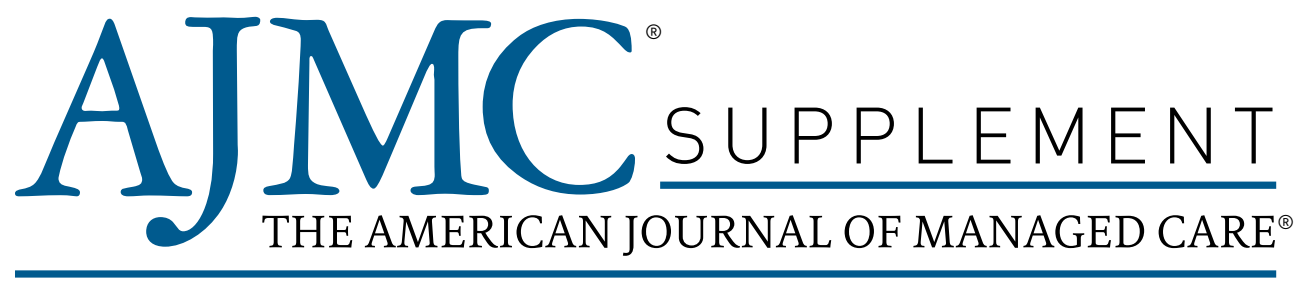

\title{
Addressing Unmet Needs in
}

\section{Alzheimer Disease: Implications of \\ Delayed Diagnosis and Examining \\ New and Emerging Therapies}

\section{HIGHLIGHTS}

> Current and Evolving Treatment Strategies for the Alzheimer Disease Continuum

> Economic Burden of Alzheimer Disease and Managed Care Considerations

> CE Sample Posttest 


\section{Addressing Unmet Needs in Alzheimer Disease: Implications of Delayed Diagnosis and Examining New and Emerging Therapies}

Release date: August 7, 2020

Expiration date: August 7, 2021

Estimated time to complete activity: 2.5 hours

Type of activity: Application

Medium: Print with internet-based posttest, evaluation, and request for credit

Fee: Free

This activity is supported by an educational grant from Biogen.

\section{Intended Audience}

Pharmacists and managed care professionals

\section{Activity Overview}

Alzheimer disease (AD) is the sixth leading cause of death and the most common etiology for dementia. It causes a significant burden to the public health system, patients afflicted with it, and their caregivers. Continuing professional education will increase competency on $\mathrm{AD}$, including the importance of early detection, patient and caregiver education, and the role of new therapeutic targets for the treatment of $\mathrm{AD}$ Application of knowledge will improve clinical decision making, improve quality of life among patients and caregivers, optimize medication therapy, improve outcomes, and decrease costs associated with the treatment of $\mathrm{AD}$.

\section{Statement of Educational Need}

Alzheimer disease requires a multispecialty approach to diagnosis and treatment. The estimated cost of Alzheimer disease includes direct medical, indirect, and social care costs. Early diagnosis can improve quality of life for both patients and caregivers. Continuing education on the importance of early diagnosis, current and emerging therapies, and the societal and economic burden of Alzheimer disease will help managed care professionals provide timely and appropriate care to patients.

\section{Educational Objectives}

Upon completion of this activity, participants should be able to:

- Explore the impact of Alzheimer disease as it relates to progression, value of early diagnosis, associated comorbidities, and effect on patients and caregivers.

- Analyze current and emerging data for new therapeutic targets for the pharmacologic treatment of Alzheimer disease.

- Examine the importance of medication therapy management and drug utilization reviews for controlling costs and improving outcomes among patients with Alzheimer disease.

\section{Accreditation Statement}

网 Pharmacy Times Continuing Education ${ }^{\mathrm{TM}}$ is accredited by the Accreditation Council for Pharmacy Education (ACPE) as a provider of continuing pharmacy education. This activity is approved for 2.5 contact hours $(0.25 \mathrm{CEU})$ under the ACPE universal activity number 0290-0000-20-207-H01-P. The activity is available for CE credit through August 7, 2021.

Obtaining Credit: Participants must read the article, complete the online posttest and an online evaluation and request for credit. Detailed instructions on obtaining CE credit are included at the end of this activity.

This CE activity is also offered free online at www.ajmc.com/ce and at www.PharmacyTimes.org/go/early-AD-suppl, where you will be directed to the activity in its entirety, including the online pretest and posttest, activity evaluation, and request for credit. 


\section{Addressing Unmet Needs in Alzheimer Disease: Implications of Delayed Diagnosis and Examining New and Emerging Therapies}

\section{OVERVIEW \\ Through this supplement to The American Journal of Managed Care ${ }^{\circledR}$, managed care profes- sionals will increase their knowledge of the burden of Alzheimer disease, current and emerging therapies, and managed care considerations.}

\section{TABLE OF CONTENTS}

Participating Faculty

S166

\section{Reports}

Current and Evolving Treatment Strategies for the Alzheimer

Disease Continuum

Richard A. Marasco, BS Pharm, FASCP, BCGP, HRM

Economic Burden of Alzheimer Disease and Managed Care Considerations $\mathbf{S 1 7 7}$

Winston Wong, PharmD

CE Sample Posttest

S184 


\section{EDITORIAL \& PRODUCTION \\ Senior Vice President Assistant Editor Jeff Prescott, PharmD, RPh Jenna Geisinger Assistant Director, Content Services Angelia Szwed Scientific Directors Danielle Jamison, \\ PharmD, MS \\ Darria Zangari \\ PharmD, BCPS, BCGP \\ Senior Clinical \\ Project Managers \\ Ida Delmendo \\ Danielle Mroz, MA \\ Clinical Project \\ Managers \\ Lauren Burawski, MA \\ Ted Pigeon \\ Project Managers \\ Lindsay Caporrino \\ Andrea Szeszko \\ Editor \\ Victoria Pelletier \\ Associate Editors \\ Hayley Fahey \\ Jill Pastor \\ Amanda Thomas \\ Medical Writers \\ Amber Schilling \\ PharmD \\ Valerie Sjoberg \\ Samantha Stone, PhD \\ Copy Chief \\ Jennifer Potash \\ Copy Supervisor \\ Paul Silverman \\ Medical \& Scientific \\ Quality Review Editor \\ Stacey Abels, PhD \\ Copy Editors \\ Georgina Carson \\ Rachelle Laliberte \\ Kirsty Mackay \\ Amy Oravec \\ Creative Director, \\ Publishing \\ Melissa Feinen \\ Art Director \\ Julianne Costello}

\section{SALES \& MARKETING}

Vice President

Gil Hernandez

Senior National

Account Managers

Ben Baruch

Megan Halsch

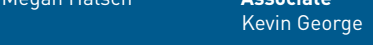

\section{OPERATIONS \& FINANCE}

Circulation Director

Jon Severn

Vice President,

Finance

Leah Babitz, CPA

Controller

Katherine Wyckoff

\section{CORPORATE}

Chairman \& Founder

Mike Hennessy Sr

Vice Chairman

Jack Lepping

President \& CEO

Mike Hennessy J

Chief Financial Officer

Neil Glasser, CPA/CFE

Executive Vice

President, Operations

Tom Tolvé

Executive Vice

President, Global

Medical Affairs \&

Afairs \&

Corporate Development

Joe Petroziello

Senior Vice President,

Content

Silas Inman

Copyright $\odot 2020$ by Managed Care

\& Healthcare Communications, LLC

AN 1 life sciences" BRAND circulation@mihassoc.com

\section{FACULTY}

Richard A. Marasco, BS Pharm, FASCP BCGP, HRM

Clinical Instructor of Pharmacy Practice

Philadelphia College of Osteopathic Medicine

Mercer University College of Pharmacy

South University College of Pharmacy

Quitman, Georgia

\section{MEDICAL WRITING \& EDITORIAL SUPPORT}

Jill E. Allen, PharmD, BCPS

Medical Writer

Pin Oak Associates

Salt Lake City, Utah

Elenna M. Smalley, PharmD, BCPS

Medical Writer

Woodbridge, Virginia

\section{FACULTY DISCLOSURES}

Richard A. Marasco, BS Pharm, HRM, and Winston Wong, PharmD, have no relevant financial relationships with commercial interests to disclose.

\section{EDITORIAL SUPPORT DISCLOSURES}

Jill E. Allen, PharmD, BCPS, and Elenna M. Smalley, PharmD, BCPS, have no relevant financial relationships with commercial interests to disclose.
Pharmacy Times Continuing Education ${ }^{\mathrm{TM}}$ Planning Staff: Jim Palatine, RPh, MBA; Maryjo Dixon, RPh; Kimberly Simpson, PharmD; Crissy Wilson; Susan Pordon; and Brianna Winters have no relevant financial relationships with commercial interests to disclose.

\section{DISCLOSURE POLICY}

According to the disclosure policy of The American Journal of Managed Care ${ }^{\circledR}$ and Pharmacy Times Continuing Education ${ }^{\mathrm{TM}}$, all persons who are in a position to control content are required to disclose any relevant financial relationships with commercial interests. If a conflict is identified, it is the responsibility of Pharmacy Times

\section{DISCLOSURE OF UNAPPROVED/OFF-LABEL USE}

The contents of this activity may include information regarding the use of products that may be inconsistent with or outside the approved labeling for these products in the United States. Participants should note that the use of these products outside current approved labeling is considered experimental and they are advised to consult prescribing information for these products.

The information provided in this CE activity is for continuing medical and pharmacy education
Continuing Education ${ }^{\mathrm{TM}}$ to initiate a mechanism to resolve the conflict(s). The existence of these relationships is not viewed as implying bias or decreasing the value of the activity. All educational materials are reviewed for fair balance, scientific objectivity of studies reported, and levels of evidence.

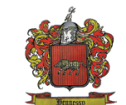

Signed disclosures are on file at the office of The American Journal of Managed Care ${ }^{\circledR}$, Cranbury, New Jersey. 


\title{
Current and Evolving Treatment Strategies for the Alzheimer Disease Continuum
}

\author{
Richard A. Marasco, BS Pharm, FASCP, BCGP, HRM
}

\section{Introduction}

Alzheimer disease (AD) is the most common cause of dementia, accounting for $60 \%$ to $80 \%$ of cases. ${ }^{1}$ It is a heterogeneous and complex disease that is challenging to differentiate from other forms of dementia, such as vascular dementia, dementia with Lewy bodies, mixed dementia, and frontotemporal dementia. Understanding of AD has evolved over the past 35 years. Before 2011, it was a clinical diagnosis that could only be confirmed by an autopsy. ${ }^{2}$ Then, diagnostic criteria were revised to allow a premortem diagnosis based on biomarkers. ${ }^{3,4}$ Now, $\mathrm{AD}$ is considered to be a long, degenerative process with a preclinical stage in which $\beta$-amyloid $(A \beta)$ and tau biomarkers are present while cognition is normal, followed by neurodegeneration and a prodrome of mild cognitive impairment (MCI), which can progress to clinical AD. ${ }^{5}$

Drug therapy that changes the progression of the disease is arguably the greatest unmet need of patients with AD. Despite investing billions of dollars into clinical trials, a new AD drug has not been approved by the FDA since $2003 .{ }^{6,7}$ Drugs approved before that do not slow the progression of the disease. ${ }^{8}$ In addition, no drugs have been approved to treat MCI. ${ }^{9}$ As of May 2020, more than 100 new molecular entities were in clinical development for AD. ${ }^{10}$ Although many AD drugs have failed in late-stage clinical trials, there is hope that at least one may receive FDA approval soon. This article explores the current state of biomarker-driven drug development across the AD continuum and reviews current and emerging disease-modifying treatments for AD.

\section{The Epidemiologic Burden of AD}

The estimated number of Americans with preclinical AD based on evidence of brain $A \beta$ is expected to rise from almost 47 million in 2017 to over 75 million by $2060 .{ }^{11}$ Modeling studies estimate that about $35 \%$ of people older than 60 years have preclinical AD. ${ }^{2}$ Because the mean time from $A \beta$ appearance to dementia is estimated at more than 20 years, some people are likely to die of other causes before developing AD. ${ }^{2,12}$ The lifetime risk of developing AD varies according to age and disease state. For example, the lifetime risk for a 75 -year-old woman is approximately $14 \%$ with no evidence

\section{ABSTRACT}

The burden of Alzheimer disease (AD) on the US healthcare system is substantial and increasing. AD progresses along a continuum from preclinical disease characterized by normal cognition and abnormal brain biomarkers to mild cognitive impairment and then clinically apparent dementia. Diagnosis early in the AD continuum has benefits for patients and caregivers and appears cost-effective, but often, the clinical diagnosis of AD may be delayed. Currently available biomarkers include $\beta$-amyloid positron emission tomography and cerebrospinal fluid tests. Collectively, they are expensive, may lead to adverse effects, are not widely available, and are not suited for primary care. Currently available treatment options, cholinesterase inhibitors and memantine, do not alter disease progression, but can help with some symptoms. Benefits of currently available treatments on cognition are difficult to quantify and are offset by a burden of adverse effects that often go unrecognized. More accurate diagnostic biomarkers and disease-modifying drug therapies are critical unmet needs of patients with $A D$ despite decades of clinical research. Because many phase 3 clinical trials that enrolled patients with symptomatic AD have failed, researchers believe that disease-modifying treatment is more likely to demonstrate benefit when utilized early in the disease continuum. Within the past few years, significant achievements that will advance clinical trials in early AD include the Research Framework to define and stage the AD continuum, FDA guidance on study design in early $A D$, and development of scales to measure cognition that are suitable for early AD. In October 2019, the AD community was re-invigorated by unexpected news that a Biologics License Application will be submitted for aducanumab to treat AD. This article explores the current state of biomarker-driven drug development across the $A D$ continuum and reviews investigational drugs in phase $2 / 3$ clinical development for AD.

Am J Manag Care. 2020;26:S167-S176

For author information and disclosures, see end of text. 
of $\mathrm{AD}$ pathology, $24 \%$ with $\mathrm{A} \beta, 36 \%$ with $\mathrm{A} \beta$ and neurodegeneration; and $85 \%$ with $A \beta$, neurodegeneration, and MCI. ${ }^{12}$ About 15 million Americans are estimated to have MCI. ${ }^{13}$ The prevalence of MCI increases with age, with estimates of $6.7 \%$ for ages $60-64$ years, $8.4 \%$ for $65-69$ years, $10.2 \%$ for $70-74$ years, $14.8 \%$ for $75-79$ years, $25.2 \%$ for $80-84$ years, and $37.6 \%$ for 85 years and older. ${ }^{9}$

Approximately $10 \%$ of Americans aged 65 years or older have AD. ${ }^{1}$ Among almost 6 million Americans 65 years or older living with $\mathrm{AD}, 80 \%$ are 75 years or older and more than $60 \%$ are women. Prevalence increases progressively with age, with AD estimates of $3 \%$ for ages $65-74,17 \%$ for ages $75-84$, and $32 \%$ for 85 years and older. By 2050, the number of Americans with AD is expected to rise to almost 14 million. The total annual cost of healthcare of patients with dementia, including long-term care and hospice, is projected to increase nearly 4-fold from $\$ 305$ billion in 2020 to more than $\$ 1.1$ trillion in 2050. ${ }^{1}$ A recent Centers for Disease Control and Prevention study identified 2 key factors that account for the rising prevalence of $\mathrm{AD}$ and other forms of dementia in the United States: a doubling in the number of Americans 65 years or older by 2060 and faster growth in minority elder populations who are disproportionately affected by dementia. ${ }^{14}$ In 2020, approximately 491,000 Americans 65 years or older are expected to receive an $\mathrm{AD}$ diagnosis and 700,000 are expected to die with $\mathrm{AD}$. $\mathrm{AD}$ is the fifth leading cause of death in Americans aged 65 years or older. ${ }^{1}$

\section{The AD Continuum}

Pathophysiology. AD is characterized by brain tissue abnormalities that are both diagnostic biomarkers and targets for drug development: accumulation of $A \beta$ plaques and oligomers outside of neurons and twisted strands of tau proteins inside neurons. ${ }^{1,6}$ In theory, $A \beta$ accumulation contributes to neurodegeneration by interfering with neuron-to-neuron synaptic communication, whereas tau tangles may block the transport of nutrients and other essential molecules within neurons. In addition, $A \beta$ and tau tangles may trigger microglia, which induce inflammation and are associated with abnormal glucose metabolism. ${ }^{15} \mathrm{AD}$ is associated with distinct abnormalities in glycolysis, the main route of glucose metabolism in the brain. ${ }^{16}$ Impaired glycolysis has been correlated with more severe $\mathrm{A} \beta$ plaque and tau tangles postmortem. ${ }^{17}$

In 2018, the National Institute on Aging and Alzheimer's Association developed the Alzheimer's Diagnostic Framework to define and classify the $\mathrm{AD}$ continuum and distinguish it from non-AD causes of cognitive impairment based on biomarker criteria. ${ }^{5}$ The hallmark biomarkers of AD pathology-extracellular deposition of $A \beta$ (“A"), intracellular tau tangles (“T”), and neurodegeneration (" $\mathrm{N}$ ")-were integrated into the " $\mathrm{A} / \mathrm{T} / \mathrm{N}$ " classification scheme to categorize subjects in $\mathrm{AD}$ clinical trials. The preclinical phase of the disease after $A \beta$ deposition is classified as A+T-N-, and the transition to prodromal disease and dementia is characterized by the addition of $\mathrm{T}$ and $\mathrm{N}$. Patients with dementia symptoms, tangles, and neurodegeneration biomarkers are classified as $\mathrm{A}+\mathrm{T}+\mathrm{N}-$ or $\mathrm{A}+\mathrm{T}-\mathrm{N}+{ }^{5}$

Apolipoprotein E (APOE) is a susceptibility gene for AD. The APOE4 allele is linked to a higher risk of AD, while the APOE2 allele is associated with a lower risk of $\mathrm{AD}$. In a recent case-control study in more than 5000 people, the odds ratio for developing $\mathrm{AD}$ with the APOE2/2 genotype was $66 \%$ less than the APOE2/3 genotype, $87 \%$ less than the APOE3/3 genotype, and $99.6 \%$ less than the APOE4/4 genotype. ${ }^{18}$ Because the relative risk conferred by APOE is impacted by gender and other genetic and environmental factors, the APOE4 allele is neither necessary nor sufficient to cause AD. APOE genotyping for $\mathrm{AD}$ risk prediction is not recommended because of limited clinical utility and poor predictive value. ${ }^{19}$ The APOE allele is a consideration in clinical trials of monoclonal antibodies and other drugs that target $A \beta$ plaque because they can cause amyloidrelated imaging abnormalities (ARIA) that can be accompanied by adverse effects (AEs). ${ }^{20,21}$

Disease course. $\mathrm{AD}$ progresses along a continuum with 3 phases: preclinical disease, MCI, and clinically apparent dementia. ${ }^{1,5,22}$ Although both cognitive decline and biomarker measurements progress over time, biomarker progression begins before symptom onset. ${ }^{5}$

Preclinical AD. The preclinical phase of the AD continuum is characterized by $A \beta$ pathology that can be detected by neuroimaging and cerebrospinal fluid (CSF) biomarkers in individuals who are not cognitively impaired. ${ }^{5}$ In longitudinal observational studies, brain accumulation of $A \beta$ in cognitively normal individuals has been linked to a greater risk of progression to MCI and dementia. ${ }^{23-25}$ Although preclinical AD is considered asymptomatic, screening data from the Anti-Amyloid Treatment in Asymptomatic Alzheimer Disease (A4) Study recently showed that participants with elevated $A \beta$ had lower Preclinical Alzheimer Cognitive Composite scores and more reports of subtle recent declines in daily cognitive function. ${ }^{26}$ It is important to bear in mind that the presence of $A \beta$ plaque does not necessarily predict the development of dementia. ${ }^{1}$

MCI. In addition to the presence of biomarkers, patients with MCI have subtle problems with memory and thinking that may not be readily apparent or interfere with the ability to perform activities of daily living (ADLs). ${ }^{1} \mathrm{MCI}$ can improve, remain stable, or progress to $\mathrm{AD}$ or other conditions. In observational studies, $32 \%$ to $38 \%$ of patients with MCI progressed to dementia over 5 years of follow-up. ${ }^{27,28}$ The American Academy of Neurology recommends evaluating patients for MCI if they or a close contact raise concerns about memory or impaired cognition. ${ }^{9}$ Early diagnosis gives patients and significant others more time to address finances and estate planning, take steps to prevent exploitation, plan for care, and address driving safety issues. ${ }^{29}$ Early diagnosis can also improve healthcare delivery. ${ }^{29}$ For example, patients may need help from a family member and written instructions to maintain medication adherence. Color-coded weekly or monthly adherence packaging prepared by the pharmacy 
can also help. Drugs with cognitive AEs that can exacerbate MCI can be avoided. Patients with MCI should be periodically monitored for changes in cognitive status. ${ }^{9}$ Documenting the diagnosis in health records alerts other clinicians and may reduce mismanagement.

Alzheimer dementia. AD severity is characterized as mild, moderate, or severe based primarily on the ability to perform ADLs. In mild disease, most patients are able to function independently but are likely to require assistance. These patients may still be able to drive and work. In moderate $\mathrm{AD}$, patients may have difficulties communicating and performing ADLs, such as bathing and dressing. They may exhibit personality and behavior changes. In severe $\mathrm{AD}$, patients need substantial assistance with ADLs and likely require around-the-clock care. These patients may become bedridden due to damage to areas of the brain involved in movement. They may have difficulty swallowing, which can lead to aspiration pneumonia, a contributing cause of death among many patients with $\mathrm{AD}$. In people aged 65 years or older who receive a diagnosis of $A D$, mean survival is 4 to 8 years, but can reach 20 years. ${ }^{1}$ A person living with $\mathrm{AD}$ between age 70 and 80 years is expected to have severe dementia for 4 years and reside in a nursing home for most of that time. ${ }^{1}$ About $40 \%$ of patients with $\mathrm{AD}$ have severe dementia that requires care on par with that provided in a nursing home. ${ }^{11}$

\section{Diagnosis}

Despite the need for early and accurate diagnosis, an estimated $29 \%$ to $76 \%$ of patients with dementia in primary care are undiagnosed. ${ }^{30}$ Clinical criteria for $\mathrm{AD}$ include a history of cognitive decline with a gradual onset and progressive course, exclusion of other causes, and documented cognitive impairment in at least one domain (complex attention, executive function, learning and memory, language, perceptual motor function, and social cognition). ${ }^{1}$ The diagnosis should be based on a thorough cognitive and neurologic examination and ideally should include history from close contacts about the patient's cognitive status. ${ }^{1}$ The diagnostic evaluation may also include blood tests and magnetic resonance imaging to document neurodegeneration and rule out other forms of dementia and neurodegenerative diseases. Batteries of neuropsychological testing may have value, including to distinguish between dementia subtypes, but they are time consuming and may not be widely available. ${ }^{31}$

Although it has been part of the Medicare Annual Wellness Visit since 2011, a recent Alzheimer's Association survey found that just $47 \%$ of primary care physicians routinely assess older patients for cognitive impairment. ${ }^{1}$ Evaluation should include direct observation of cognitive function and may include a brief validated, structured cognitive assessment tool. A positive cognitive screening test should lead to additional diagnostic testing to confirm the diagnosis and determine the dementia subtype. ${ }^{30}$
Of note, a 2020 US Preventive Services Task Force panel found insufficient evidence to recommend for or against routine cognitive impairment screening in asymptomatic community-dwelling adults 65 years old and older. ${ }^{30}$

Cognitive screening tests. Many standardized mental status scales are used to document the presence and progression of dementia, including the Montreal Cognitive Assessment and the Mini-Mental State Examination (MMSE). Although these scales can accurately differentiate clinically apparent Alzheimer-type disease (CATD) from normal cognition, they are less accurate in distinguishing mild CATD versus normal cognition and CATD versus MCI. ${ }^{32}$

Biomarkers. More accurate diagnostic biomarkers are a critical unmet need because $15 \%$ to $30 \%$ of patients with CATD do not meet postmortem diagnostic criteria for AD. ${ }^{1}$ Current diagnostic biomarkers that differentiate $\mathrm{AD}$ from other forms of dementia include brain imaging and CSF biomarkers. FDA-approved $\mathrm{A} \beta$ positron emission tomography (PET) scanning agents include florbetapir, flutemetamol, and florbetaben. The Alzheimer's Association and Society of Nuclear Medicine and Molecular Imaging recommend use by dementia experts evaluating patients with cognitive impairment if scan results will improve diagnostic certainty and alter the treatment plan. ${ }^{33}$ A recent Agency for Healthcare Research and Quality systematic review found that A $\beta$ PET was highly sensitive and specific for $\mathrm{A} \beta$ pathology of $\mathrm{AD}$ and may increase classification accuracy. ${ }^{31}$ In clinical trials evaluating disease-modifying drugs for $\mathrm{AD}$, biomarkers play a crucial role in identifying eligible patients and contribute to the high cost of AD clinical trials. The out-of-pocket cost per scan is at least $\$ 3000 .^{34}$ Although commercial insurance coverage varies, Medicare does not cover them unless they are conducted as part of a clinical trial assessing whether $A \beta$ imaging improves patient outcomes or advances patient treatment options. ${ }^{34}$

The Imaging Dementia-Evidence for Amyloid Scanning (IDEAS) study is evaluating whether A $\beta$ PET scanning affects the management and outcomes of dementia care in Medicare beneficiaries. ${ }^{35}$ In the initial phase of the study, physicians changed treatment based on scan results in greater than $60 \%$ of patients. More than a third of patients who were presumed to have $\mathrm{AD}$ had a negative PET scan, whereas more than half of patients presumed not to have $\mathrm{AD}$ had a positive PET scan. In patients with a positive PET scan, AD drug prescribing increased from $40 \%$ to $82 \%$ in patients with $\mathrm{MCI}$ $(P<.001)$, although no drug has established efficacy to treat MCI. ${ }^{9}$ Prescribing increased from $63 \%$ to $91 \%$ in patients with dementia $(P<.001)$. AD drug prescribing decreased modestly in patients with a negative scan. ${ }^{35}$

The first diagnostic imaging agent that identifies AD-associated tau pathology may be available soon. The FDA is considering approval of flortaucipir, a PET scanning tracer that binds with tau tangles. ${ }^{36}$ In a phase 3 postmortem validation study, it had sensitivity of $92 \%$ to $100 \%$ and specificity of $52 \%$ to $92 \%$ for predicting tau pathology. ${ }^{37}$ 
PET and CSF biomarkers for $A \beta$ are invasive, expensive, time consuming, cause AEs, and are not widely available. ${ }^{13,34}$ Biomarkers in clinical development may address these limitations. For example, a blood test for plasma phosphorylated-tau181 predicted tau and $A \beta$ pathologies, identified $A D$ across the clinical continuum, and differentiated it from other neurodegenerative diseases in validation studies. ${ }^{38,39}$

\section{AD Management Considerations}

Public health burden. AD is a substantial public health burden because patients are disabled and dependent on others for care for a substantial portion of the disease. ${ }^{1}$ The disease burden of $\mathrm{AD}$ has increased dramatically in the United States over the past few decades. Disability-adjusted life-years (DALYs), the primary measure of disease burden, are calculated by adding the number of years of life lost due to premature mortality (YLLs) and the number of years lived with disability (YLDs) caused by a disease. Based on DALYs, AD rose in the ranking of burdensome diseases from twelfth in 1990 to sixth in 2016. In 2016, it ranked fourth for YLLs and nineteenth for YLDs. ${ }^{1}$

Value of early diagnosis. A recent study commissioned by the Alzheimer's Association estimated the potential cost savings of early diagnosis. ${ }^{1}$ Diagnosis in the MCI phase rather than in the dementia phase or not at all could save approximately $\$ 7$ trillion in medical and long-term care costs. Cost savings would accrue from a lower cost to diagnose MCI versus dementia and lower medical and long-term care costs for diagnosed and managed MCI and dementia versus unmanaged MCI and dementia. ${ }^{1}$ Unfortunately, many barriers exist to early diagnosis of MCI in primary care, including a lack of assessment tools, training, time, and infrastructure. ${ }^{40}$

Comorbidities. Medicare beneficiaries with dementia have a higher rate of comorbidities than those without dementia. ${ }^{1}$ For example, the prevalence of more than 5 chronic conditions is $26 \%$ and $4 \%$, respectively. In 2014, among Medicare beneficiaries with dementia, 38\% had comorbid coronary artery disease, $37 \%$ had diabetes, $29 \%$ had chronic kidney disease, $28 \%$ had congestive heart failure, and $25 \%$ had chronic obstructive pulmonary disease. ${ }^{1}$

Caregiver burden. As the US population ages, the proportion of younger adults available to provide care for patients with $\mathrm{AD}$ is expected to decline. ${ }^{14}$ Whereas the ratio of potential younger caregivers to older high-risk adults is currently 7 to 1 , it is expected to decline to 4 to 1 by 2030. Negative impacts on caregivers include greater work instability, lower household income and personal savings, increased food insecurity, and less personal medical care. This leads to caregiver health problems, higher rates of depression and psychological stress, and even a higher risk of mortality. ${ }^{1,14}$ In 2017, Medicare implemented a billing code to reimburse services related to care planning and coordination for patients with cognitive impairment and their caregivers. This is intended to provide greater caregiver support. ${ }^{1}$
The COVID-19 pandemic has created a cascade of negative effects for patients with dementia and their caregivers. It has amplified the burden of caregiving for patients with dementia. Mortality from COVID-19 is extremely high in patients with dementia due to advanced age, comorbidities, and difficulty complying with safeguarding procedures such as wearing masks, handwashing, and social distancing. ${ }^{41,42}$ COVID-19 outbreaks in nursing homes have high attack and case fatality rates. ${ }^{42,43}$ Whereas nursing home residents and workers account for $11 \%$ of COVID-19 cases in the United States, they account for $35 \%$ of deaths. ${ }^{43}$ Given this risk, facilities have implemented numerous restrictions. For patients who are cared for at home, social distancing measures and the loss of in-home health aides and adult day care has placed the entire burden of caregiving on immediate family members. ${ }^{41}$ Caregivers, whether at home or in nursing homes, are experiencing extreme stress and social isolation during the pandemic. ${ }^{41,42}$

\section{Current Management of the AD Continuum}

Patients on the AD continuum often take medications that impair cognition and may accelerate the disease trajectory, including benzodiazepines, anticholinergics, and other psychotropic drugs. ${ }^{44-46}$ Because "Do no harm" is a guiding precept in elder care, avoiding these drugs is a crucial strategy to preserve cognition. ${ }^{47}$ The Beers list of potentially inappropriate medications acts as a guide to avoiding harmful prescribing in patients with $\mathrm{AD} .{ }^{48}$ Given that medication nonadherence is endemic in patients with $\mathrm{AD}$, it is important to simplify drug regimens and deprescribe unnecessary drugs. ${ }^{49}$ Factors linked to medical nonadherence in patients with dementia include taking more than 4 drugs, use of an anticholinergic, and a pill burden. ${ }^{49}$ Caregivers should receive medication management support, with periodic reassessment of the patient's medication needs as AD progresses. ${ }^{50} \mathrm{~A}$ randomized clinical trial is currently evaluating whether deprescribing inappropriate medications and optimizing medication regimens can delay the onset of symptomatic AD and other forms of dementia. ${ }^{51}$

Nonpharmacologic management of AD. A few nondrug modalities have been shown to improve cognition in patients with AD. Exercise has positive effects on cognitive function, and may even slow the rate of cognitive decline in patients with $\mathrm{AD} .{ }^{1}$ Cognitive stimulation has been found to improve cognitive function, depression, anxiety and quality of life (QOL) in patients with $\mathrm{AD}$. Cognitive training may improve overall cognition that may last for at least a few months in patients with mild to moderate dementia. ${ }^{1}$ Nondrug modalities also play an important role in reducing behavioral symptoms, such as sleep disturbances, wandering, depression, agitation, and aggression. Nonpharmacologic interventions for agitation and aggression avoid the potential harms of antipsychotics and may outperform pharmacologic approaches. For example, outdoor activities were found to be more efficacious than antipsychotics 
for managing physical aggression. ${ }^{52}$ Other beneficial modalities for agitation include multidisciplinary care planning, pet therapy, music, and massage. ${ }^{52}$

Pharmacologic management of AD. As shown in Table 1, FDA-approved drugs for AD include cholinesterase inhibitors and memantine. ${ }^{53}$ Cholinesterase inhibitors increase levels of the neurotransmitter acetylcholine while memantine is an N-methylD-aspartate (NMDA) receptor antagonist that affects glutamatergic transmission. Memantine is used alone or in combination with a cholinesterase inhibitor. Each of these medications is taken orally, once or twice daily. Rivastigmine is also available as a transdermal patch that is applied once daily. ${ }^{53}$

Cholinesterase inhibitors and memantine do not change the progression of AD. In the 1-year AD2000 clinical trial, donepezil demonstrated slight improvements in cognition and function, but failed to delay institutionalization, reduce caregiver burden, or lower costs. ${ }^{54}$ Results of a recent Cochrane review of donepezil trials showed a mean reduction of -2.7 points on the Alzheimer's Disease Assessment Scale-Cognitive (ADAS-Cog, range 0-70), but no benefit for behavioral symptoms, QOL, or total healthcare resource utilization. ${ }^{55}$ This falls below the 4-point difference on the ADAS-Cog that is generally recognized as clinically meaningful. ${ }^{56}$ AEs with donepezil were dose related. In particular, donepezil $23 \mathrm{mg} /$ day did not improve cognitive function more than $10 \mathrm{mg} /$ day, but increased the risk of AEs and premature treatment discontinuation. ${ }^{55}$ In another recent Cochrane review, memantine demonstrated a small clinical benefit versus placebo in patients with moderate to severe $A D$, but had no benefit in patients with mild AD. ${ }^{57}$

In a systematic review that excluded clinical trials with a high risk of bias, cholinesterase inhibitors and memantine slightly reduced short-term cognitive decline. ${ }^{8}$ It was unclear if these effects are clinically meaningful. Cholinesterase inhibitors had a small mean improvement in cognition with a median standardized mean difference (SMD) of 0.30 (range, 0.24, 0.52). For function, effects ranged from no difference to a small improvement, with a median SMD of 0.19 (range, -0.10-0.22). Memantine did not demonstrate any benefit in patients with mild to moderate CATD. In moderate to severe CATD, insufficient to low-strength evidence inconsistently suggested that adding memantine to a cholinesterase inhibitor improved cognition, but not function. ${ }^{8}$

As part of the Choose Wisely initiative, the American Geriatrics Society recommends against prescribing cholinesterase inhibitors unless cognitive benefits and gastrointestinal AEs are monitored. ${ }^{58}$ A cholinesterase inhibitor should be stopped after 12 weeks if improvements are not observed on practical treatment outcomes reflecting stabilization of cognition that can be easily assessed. Patients and caregivers should be counseled about AEs before beginning a trial of cholinesterase inhibitors. Deprescribing of cholinesterase inhibitors has been linked to a reduction in falls and fractures, but not an increase in the risk of aggressive behaviors and antipsychotic prescribing in nursing home residents with severe dementia. ${ }^{59,60}$ Cholinesterase inhibitor AEs are often unrecognized and lead to a "prescribing cascade" that contributes to a treatment burden in patients with AD. For example, observational studies have found that patients receiving a cholinesterase inhibitor had an increased risk of receiving an anticholinergic drug to manage urinary incontinence, which is likely caused or exacerbated by the cholinesterase inhibitor. ${ }^{61,62}$

\section{Treatment Landscape of Emerging Agents for the AD Continuum}

Since 2007, more than 50 drugs have failed in phase 3 clinical trials for $\mathrm{AD} .^{63}$ This includes $\mathrm{A} \beta$-targeting agents (bapineuzumab, crenezumab, semagacestat, solanezumab), $\beta$-secretase inhibitors (elenbecestat, lanabecestat, umibecestat, verubecestat), intravenous immunoglobulin, aspirin, ginkgo biloba, idalopirdine, minocycline,

TABLE 1. FDA-approved Drugs for Alzheimer Disease $\mathrm{s}^{53}$

\begin{tabular}{|c|c|c|c|}
\hline Drug & AD Indications & Dosing & Common Adverse Effects \\
\hline $\begin{array}{l}\text { Donepezil } \\
\text { (Aricept) }\end{array}$ & All stages & Once daily in the evening & $\begin{array}{c}\text { Nausea, vomiting diarrhea, urinary incontinence, vivid } \\
\text { dreams, bradycardia, syncope }\end{array}$ \\
\hline $\begin{array}{l}\text { Galantamine } \\
\text { (Razadyne) }\end{array}$ & $\begin{array}{l}\text { Mild to } \\
\text { moderate }\end{array}$ & $\begin{array}{l}\text { Tablets: twice daily } \\
\text { Extended release capsules: } \\
\text { once daily }\end{array}$ & $\begin{array}{l}\text { Nausea, vomiting, diarrhea, dizziness, anorexia, weight } \\
\text { loss, bradycardia, syncope }\end{array}$ \\
\hline $\begin{array}{l}\text { Rivastigmine } \\
\text { (Exelon Patch) }\end{array}$ & All stages & $\begin{array}{l}\text { Capsules: twice daily } \\
\text { Patches: applied once daily }\end{array}$ & $\begin{array}{l}\text { Oral: nausea, vomiting, diarrhea. } \\
\text { Patch: gastrointestinal adverse effects less frequent. } \\
\text { Bradycardia, syncope with oral or patch }\end{array}$ \\
\hline $\begin{array}{l}\text { Memantine } \\
\text { (Namenda and Namenda XR) }\end{array}$ & $\begin{array}{l}\text { Moderate to } \\
\text { severe }\end{array}$ & Once daily & $\begin{array}{l}\text { Generally, well tolerated; can cause dizziness, confusion, } \\
\text { agitation, insomnia, hallucinations, delusions }\end{array}$ \\
\hline $\begin{array}{l}\text { Memantine + donepezil } \\
\text { (Namzaric) }\end{array}$ & $\begin{array}{l}\text { Moderate to } \\
\text { severe }\end{array}$ & Once daily in the evening & See components \\
\hline
\end{tabular}


nivaldipine, pioglitazone, and others. ${ }^{6,64-77}$ Because these failed studies enrolled patients with symptomatic $\mathrm{AD}$, many researchers now believe that clinical trials of disease-modifying drugs should be conducted much earlier, either during the preclinical or MCI phases of the AD continuum. ${ }^{1}$ In 2018, the FDA published a draft guidance for drug development in early AD that aligns with this thinking. ${ }^{78}$ As shown in Table 2 , it does away with the preclinical/ prodromal definition in favor of 3 stages leading to early $\mathrm{AD} .{ }^{78} \mathrm{It}$ now refers to a "persistent effect on disease course" instead of a disease-modifying effect. ${ }^{79}$

Many factors may have contributed to the high failure rate of $\mathrm{AD}$ phase 3 trials. Because $\mathrm{AD}$ is a heterogeneous disease, the inaccuracy of disease biomarkers is a likely factor. ${ }^{7}$ In hindsight, some trials may have failed because dose response was poorly understood. ${ }^{80}$ Now experts assert that before advancing to phase 3 trials, a drug should demonstrate sufficient brain exposure, strong evidence of target engagement, a clear dose response, and a large effect size in phase 2 trials. ${ }^{21,80,81}$ Target engagement is a hot-button issue for investigational anti-A $\beta$ monoclonal antibodies. Some experts theorize that some have failed because they target amyloid monomers or insoluble fibrils or plaques rather than soluble $A \beta$ oligomers, which may be a key mediator of neurodegeneration. ${ }^{21}$ Coprimary study end points of cognition and function that were required by the FDA may have also contributed by setting an unachievable standard for efficacy. ${ }^{7}$ The draft guidance now suggests an AD biomarker and a cognitive or functional end point could be used to demonstrate efficacy in the earliest stages of AD (FDA stage 1 and 2). ${ }^{7}$ It also signals that the agency is willing to grant accelerated approval based on biomarker data with a requirement that a confirmatory clinical trial demonstrates a delay in symptom onset. ${ }^{\text {? }}$

Up to now, clinical trials have used the ADAS-cog as an efficacy end point, but it is not very sensitive or reliable in mild dementia. ${ }^{7}$ For patients in FDA stage 3 criteria (MCI), the draft guidance suggests an integrated scale that assesses both function and cognitive effects could serve as a single primary efficacy end point. New instruments, such as the integrated $\mathrm{AD}$ rating scale or AD Composite Score (ADCOMS), combine cognitive and functional outcomes. ADCOMS combines portions of the ADAS-cog, Clinical Dementia Rating (CDR) scale, and MMSE that have been shown to change the most over time in people who do not have functional impairment yet. ${ }^{7}$ As of May 2020 , there were 13 drugs in phase $2 / 3$ clinical development for $\mathrm{AD} .{ }^{10,82}$ They target $\mathrm{A} \beta$, tau, inflammation, neuroprotection, and metabolism. ${ }^{75}$

\section{A $\beta$-Targeting Investigational Drugs}

Aducanumab. This fully-human IgG1 monoclonal antibody binds selectively to aggregated $\mathrm{A} \beta$ fibrils and soluble oligomers. ${ }^{83,84}$ In March 2019, after an interim futility analysis predicted the phase 3 placebo-controlled EMERGE and ENGAGE trials would not meet their primary end points, the termination of all aducanumab clinical trials was announced. However, in a subsequent analysis of a larger data set from the EMERGE trial, aducanumab met the primary end point, the Clinical Dementia Rating-Sum of Boxes (CDR-SB) score after 18 months of treatment. ${ }^{85}$ The highest dose $(10 \mathrm{mg} / \mathrm{kg})$ demonstrated a $23 \%$ reduction in the CDR-SB $(P=.01)$. This translates to an absolute change of -0.4 on an 18-point scale. The high-dose group also declined less on secondary end points, including the ADAS-Cog (-27\%; $P=.01)$ and the Alzheimer's Disease Cooperative Study-Activities of Daily Living scale (ADCS-ADL-MCI; $-40 \% ; P=.001$ ). Although the ENGAGE trial did not meet the primary end point, an exploratory analysis suggested slower decline in patients who received at least 10 doses of the highest aducanumab dose. In sub-studies of EMERGE and ENGAGE, aducanumab caused a dose-dependent reduction in $A \beta$ and some reduction in CSF phosphorylated-tau. ${ }^{85}$

Amyloid-related imaging abnormalities-edema (ARIA-E), the most common AE with aducanumab, occurred in $35 \%$ of patients; $74 \%$ of cases were asymptomatic and episodes generally resolved within 4 to 16 weeks. ${ }^{85}$ In the EMERGE trial, the rate of permanent treatment discontinuation for an AE was $2.9 \%$ with placebo versus $7.7 \%$ with low-dose and $8.8 \%$ with high-dose aducanumab. ${ }^{86}$ Respective discontinuation rates because of ARIA were $0.2 \%, 4.6 \%$, and $6.6 \%{ }^{86}$

What accounts for these divergent results? The initial analysis included about half as many patients as the final data set and was limited to patients who had completed the study by the end of 2018. These patients, early enrollers in the studies, had lower mean aducanumab exposure. The futility analysis was not adjusted to consider the effect of 2 late protocol amendments that led to more patients receiving the highest dose of aducanumab later in the study ${ }^{87}$ Specifically, these protocol amendments increased the dose in APOE4 carriers who are more susceptible to ARIA after data from other studies suggested ARIA is manageable and usually resolves without sequelae. In early July 2020, the manufacturer completed

TAB LE 2. FDA-Proposed Stages of Early $A D^{78}$

\begin{tabular}{|c|c|c|c|}
\hline Stage 1 & Stage 2 & Stage 3: MCI & Stage 4: Mild Dementia \\
\hline $\begin{array}{l}\text { - AD biomarkers present } \\
\text { - No observable cognitive or } \\
\text { functional impairment }\end{array}$ & $\begin{array}{l}\text { - Early cognitive decline } \\
\text { - No functional impairment } \\
\text { on ADLs }\end{array}$ & $\begin{array}{l}\text { - Cognitive decline } \\
\text { - Mild detectable } \\
\text { functional impairment }\end{array}$ & $\begin{array}{l}\text { - Demonstrable cognitive decline } \\
\text { - Demonstrable } \\
\text { functional impairment }\end{array}$ \\
\hline
\end{tabular}

AD, Alzheimer disease; ADL, activity of daily living; $\mathrm{MCl}$, mild cognitive impairment. 
its submission of a Biologics License Application for aducanumab as a treatment for AD. ${ }^{88,89}$

BAN2401. This humanized monoclonal antibody has greater affinity for soluble $A \beta$ protofibrils (ie, large amyloid oligomers) than insoluble fibrils or plaques. ${ }^{20}$ In Study 201, a phase $2 \mathrm{~b}$ trial, there was a significant dose-dependent reduction in A $\beta$ PET burden across all doses in 856 patients with MCI or early AD. At 18 months, $81 \%$ of participants on the highest dose had $A \beta$-negative scans. There was a dose-dependent reduction in cognitive decline on the ADCOMS, starting at 6 months. The highest dose had a $30 \%$ reduction in the ADCOM versus placebo $(P=.034)$. Dose-related ARIA-E occurred in fewer than $10 \%$ of patients and was more frequent in APOE4positive patients. It generally occurred within the first 3 months of treatment, resolved within 4 to 12 weeks, and caused headache or confusion in about $10 \%$ of cases. ${ }^{20}$

Based on Study 201 results, the phase 3 CLARITY trial is examining BAN2401 in more than 1500 patients with MCI due to AD or mild AD with confirmed $A \beta$ pathology. ${ }^{90}$ In this study, BAN2401 will be given at a dose of $10 \mathrm{mg} / \mathrm{kg}$ every other week. The primary end point is the change from baseline in the CDR-SB after 18 months of treatment. Initial results are expected in 2022. ${ }^{90}$ BAN2401 is also being evaluated for the prevention of $\mathrm{AD}$ in the placebo-controlled phase 3 AHEAD 3-45 trial. ${ }^{91,92}$ The trial consists of 2 sub-studies: A3 and A45. The A3 sub-study will evaluate low-dose BAN2401 in cognitively normal individuals who are currently below the threshold for $A \beta$ elevation on $A \beta$ PET scanning but are at high risk for further $A \beta$ accumulation. The A45 sub-study will evaluate high-dose BAN2401 in clinically normal participants (ie, little to no cognitive impairment) with elevated levels of $A \beta$ and are at high risk for progression to $\mathrm{MCI}$ and $\mathrm{AD}$ dementia. ${ }^{91,92}$

Gantenerumab. This IgG1 human monoclonal antibody binds to aggregated forms of $A \beta$ and has been shown to reduce the burden of $\mathrm{A} \beta$ plaque in patients with $\mathrm{AD} .{ }^{63,93}$ Two ongoing phase 3 clinical trials, GRADUATE 1 and 2, are assessing subcutaneous (SC) gantenerumab for the treatment of early AD in more than 2000 patients. Gantenerumab is the only anti-A $\beta$ agent in late-stage clinical trials that is administered by SC injection. In February 2020 , it was announced that gantenerumab did not meet its primary end point in the DIAN-TU trial, an international clinical trial evaluating multiple drugs in patients with or at risk for autosomal dominant AD. The GRADUATE studies are evaluating a higher gantenerumab dose that most patients received in the DIAN-TU trial. Results are expected in $2022 . .^{93}$

\section{Tau-targeting Investigational Drugs}

LMTX. This prodrug of methylene blue, a second-generation tau aggregation inhibitor, is the only tau-specific agent in phase 3 clinical trials. ${ }^{6,94}$ Blinding of LMTX in clinical trials is problematic because it discolors urine. Two phase 3 clinical trials of LMTX for mild to moderate AD (TRX-005 and TRX-015) were completed in 2016. Based on phase 2 clinical trial results, these studies compared LMTX doses of 150-250 mg/day with $8 \mathrm{mg} /$ day as an intended control that would maintain blinding. In TRX-015, $8 \mathrm{mg} /$ day appeared to show the same benefit as the higher doses, and LMTX monotherapy appeared more effective than coadministration with cholinesterase inhibitors and/or memantine. These findings led to changes in the design of the ongoing TRX-005 study. That study met modified primary prespecified outcomes and confirmed the results seen in Study TRX-015. ${ }^{95} \mathrm{~A}$ third phase 3 clinical trial, LUCIDITY, is currently underway to confirm whether low-dose monotherapy is effective. ${ }^{96}$ Top-line results are expected by the end of 2021. To maintain blinding, a urine discolorant has been added to some placebo pills in this study. ${ }^{96}$ AEs with LMTX included diarrhea, dysuria, and decreased hemoglobin. It does not appear to cause ARIA. ${ }^{6}$

\section{Inflammation-targeting Investigational Drugs}

ALZT-OP1. This combination regimen consists of nasally inhaled cromolyn, a mast cell stabilizer, and oral ibuprofen, a nonsteroidal anti-inflammatory agent. In theory, it could reduce neuroinflammation and promote clearance of $\mathrm{A} \beta$ by converting microglia from a proinflammatory to a phagocytic state. ${ }^{94}$ The phase 3 COGNITE trial is evaluating whether ALZT-OP1 slows or reverses cognitive and functional decline on the CDR-SB in patients with early AD. ${ }^{97}$

COR388. This oral small-molecule agent irreversibly inhibits gingipains. ${ }^{98}$ These are virulence factor proteases from Porphyromonas gingivalis, a periodontal pathogen that has been found in the brain of patients with AD. COR388 is being evaluated in the phase 2/3 GAIN clinical trial. ${ }^{99}$ It is evaluating COR388 given twice daily in 570 patients with mild to moderate AD. An interim analysis is expected by the end of 2020, and top-line results are expected by the end of $2021 .^{99}$

Masitinib. This selective tyrosine kinase inhibitor acts on mast cells and modulates neuroinflammation. ${ }^{100}$ Potential mechanisms of action in $\mathrm{AD}$ include modulating neuroinflammation mediated by mast cells and inhibition of Fyn, a protein kinase involved in $A \beta$ signaling and tau phosphorylation. In June 2019, interim analysis of the phase 3 AB09004 clinical trial indicated that the study should continue. The study is comparing masitinib 4.5 or $6 \mathrm{mg} / \mathrm{kg} /$ day with placebo in 720 patients with confirmed mild to moderate AD receiving a cholinesterase inhibitor and/or memantine. ${ }^{100}$ The study is expected to complete in $2020 .^{101}$

\section{Other Investigational Drugs}

AGB101. Levetiracetam is being repurposed as a synaptic vesicle glycoprotein 2A (SV2A) modulator that is hypothesized to decrease A $\beta$-induced hippocampal hyperactivity. ${ }^{75}$ The phase 3 HOPE4MCI clinical trial is evaluating low-dose extended-release levetiracetam at a dose $220 \mathrm{mg}$ once daily to slow cognitive and functional impairment, 
as in patients with MCI. ${ }^{102}$ The primary outcome is change in the CDR-SB. Top-line results are expected in September 2022. ${ }^{102}$

Blarcamesine (ANAVEX2-73). This sigma-1 receptor agonist targets protein misfolding, and may reduce tau hyperphosphorylation, oxidative stress, and neurodegeneration in $\mathrm{AD} .{ }^{103}$ In a phase 2a study, it demonstrated dose-dependent improvement in MMSE ADCS-ADL. A 48 -week phase $2 \mathrm{~b} / 3$ study in 450 patients with early $\mathrm{AD}$ is ongoing. Top-line results are expected in September 2023. ${ }^{104}$

CAD106. This second-generation active $A \beta$ vaccine contains multiple copies of the $A \beta_{1-6}$ peptide linked to a carrier containing many copies of bacteriophage $\mathrm{Qb}$ coat protein. ${ }^{105}$ In animal studies, it induced $A \beta$ antibodies without triggering an $A \beta$-specific T-cell response. It is under evaluation in a 5 -year phase $2 / 3$ trial in individuals aged 60 to 75 years who are asymptomatic APOE4 carriers with elevated $\mathrm{A} \beta$ in CSF or on PET scan. ${ }^{106} \mathrm{CAD} 106$ is given by intramuscular injection every 6 weeks for the first 3 injections and then every 3 months thereafter. The CAD106 trial is expected to yield top-line results by the end of $2024 .{ }^{107}$ In a phase $2 \mathrm{~b}$ clinical trial, serious AEs more frequent with CAD106 than placebo included headache, hypertension, and fever. Several cases of ARIA occurred in CAD106-treated patients. ${ }^{105}$

Icosapent ethyl. The phase 2/3 BRAVE-EPA study is evaluating the effect of icosapent ethyl in 150 cognitively-normal APOE4positive veterans aged 50 to 75 years. Initial results are expected in late $2021 .^{108}$

Plasma exchange. The phase 2/3 Alzheimer's Management by Albumin Replacement (AMBAR) trial assessed whether plasma exchange and infusions of albumin \pm intravenous immunoglobulin (IVIG) would delay progression of mild to moderate AD. ${ }^{109}$ Plasma exchange may remove albumin-bound $A \beta$ circulating in plasma. Albumin and IVIG have immunomodulatory and antiinflammatory properties. In top-line data, plasma exchange did not significantly affect the coprimary end point of ADAS-Cog and ADCS-ADL scores compared with placebo at 14 months but had significant benefits on some individual end points. The sponsor intends to meet with the FDA to discuss the design of a successive AMBAR II trial to confirm whether the protocol has benefit. ${ }^{110}$ The feasibility of plasma exchange, an invasive and expensive procedure, to manage progression of $\mathrm{AD}$ is questionable. ${ }^{11}$

Troriluzole (BHV-4157). This once-daily oral drug is a prodrug conjugate of riluzole that may reduce glutamate-mediated excitotoxicity and nerve cell deterioration by promoting nerve cell glutamate reuptake. ${ }^{75}$ It is being evaluated in the $\mathrm{T} 2$ Protect $\mathrm{AD}$ phase 3 clinical trial, which could have preliminary results by late $2020 .{ }^{112}$

\section{The Future of AD Drug Development}

More than 1000 clinical trials have been put on hold because of the COVID-19 pandemic. ${ }^{113}$ This includes clinical trials in AD that have enrolled participants who are especially vulnerable to COVID-19 complications. ${ }^{114}$ Researchers are concerned that data collection in ongoing $\mathrm{AD}$ clinical trials may be harmed. Because conducting clinical trials in $\mathrm{AD}$ is already a daunting undertaking, there is also concern that some pharmaceutical companies may abandon a challenging drug development area such as $\mathrm{AD}$ in this time of crisis. ${ }^{42}$

What does the future hold if a drug receives FDA approval to prevent progression of preclinical or prodromal AD? A RAND Corporation analysis suggests that a paradigm shift to preventing progression in people with preclinical or prodromal disease could create enormous challenges for the US healthcare system, especially managed care. ${ }^{13}$ Necessary resources such as dementia specialists, PET imaging facilities, and infusion centers are in short supply. Between 2020 and 2040, 2.1 million patients might develop AD while on a waiting list for treatment. ${ }^{13}$ Considering the large number of individuals with preclinical $\mathrm{AD}$, the cost of preventive therapy could be enormous. ${ }^{115}$ Assessing the value of treatment will likely be a complex undertaking if a drug is approved based on a surrogate measure (eg, cognitive test score improvement) that does not guarantee prevention of dementia. For the first time in almost 20 years, the FDA is evaluating whether to approve a drug for AD that could modify its course. This exciting news has broad implications in managed care.

Author affiliation: Richard A. Marasco, BS Pharm, FASCP, BCGP, HRM, is a clinical instructor of pharmacy practice, Philadelphia College of Osteopathic Medicine, Mercer University College of Pharmacy, South University College of Pharmacy, Quitman, GA.

Funding source: This activity is supported by an educational grant from Biogen MA, Inc.

Author disclosure: Dr Marasco has no relevant financial relationships with commercial interests to disclose.

Authorship information: Substantial contributions to the concept and design; drafting of the manuscript; overall supervision; and critical revision of the manuscript for important intellectual content.

Address correspondence to: rmarasco@seniorpharm.com

Medical writing and editorial support: Jill E. Allen, PharmD, BCPS

\section{REFERENCES}

1. Alzheimer's Association. 2020 Alzheimer's disease facts and figures. Alzheimers Dement. 2020;16(3):1-391 2. Mar J, Soto-Gordoa M, Arrospide A, Moreno-Izco F, Martínez-Lage P. Fitting the epidemiology and neuropathology of the early stages of Alzheimer's disease to prevent dementia. Alzheimers Res Ther. 2015;7(1):2. doi: 10.1186/s13195-014-0079-9

3. McKhann GM, Knopman DS, Chertkow H, et al. The diagnosis of dementia due to Alzheimer's disease: recommendations from the National Institute on Aging-Alzheimer's Association workgroups on diagnostic guidelines for Alzheimer's disease. Alzheimers Dement. 2011;7(3):263-269.

4. Albert MS, DeKosky ST, Dickson D, et al. The diagnosis of mild cognitive impairment due to Alzheimer's disease: recommendations from the National Institute on Aging-Alzheimer's Association workgroups on diagnostic guidelines for Alzheimer's disease. Alzheimers Dement. 2011;7(3):270-279. doi: 10.1016/j.jalz.2011.03.008

5. Jack CR Jr, Bennett DA, Blennow K, et al. NIA-AA Research Framework: Toward a biological definition of Alzheimer's disease. Alzheimers Dement. 2018;14(4):535-562. doi: 10.1016/j.jalz.2018.02.018

6. Yiannopoulou KG, Papageorgiou SG. Current and future treatments in Alzheimer disease: an update J Cent Nerv Syst Dis. 2020;12:1179573520907397. doi: 10.1177/1179573520907397

7. Sabbagh MN, Hendrix S, Harrison JE. FDA position statement "Early Alzheimer's disease: Developing drugs for treatment, Guidance for Industry". Alzheimers Dement (N Y). 2019:5:13-19. doi: 10.1016/j.trci.2018.11.004

8. Fink HA, Linskens EJ, MacDonald R, et al. Benefits and harms of prescription drugs and supplements for treatment of clinical Alzheimer-type dementia: a systematic review and meta-analysis. Ann Intern Med. 2020;172(10):656-668. doi: 10.7326/M19-3887

9. Petersen RC, Lopez 0, Armstrong MJ, et al. Practice guideline update summary: mild cognitive impairment: Report of the Guideline Development, Dissemination, and Implementation Subcommittee of the American Academy of Neurology. Neurology. 2018;90(3):126-135. doi: 10.1212/WNL.0000000000004826 
10. Alzforum. Therapeutics search. Published 2020. Accessed May 12, 2020. alzforum.org/therapeutics/ search?fda statuses $\% 5 \mathrm{~B} \% 5 \mathrm{D}=1818$ fda statuses $\% 5 \mathrm{~B} \% 5 \mathrm{D}=33251$ \&fda statuses $\% 5 \mathrm{~B} \% 5 \mathrm{D}=1828$ fda statuses $\% 5 \mathrm{~B} \% 5 \mathrm{D}=931 \&$ fda_statuses\% $\% \overline{\mathrm{B}} \% 5 \mathrm{D}=183 \&$ target_types=\&therapy_types=\&conditions $\% 5 \mathrm{~B} \% 5$ $\mathrm{D}=145 \&$ conditions $\% 5 \mathrm{~B} \% 5 \mathrm{D}=146 \&$ keywords-entry=\&keywords=\#results 11. Brookmeyer R, Abdalla N, Kawas CH, Corrada MM. Forecasting the prevalence of preclinical and clinical Alzheimer's disease in the United States. Alzheimers Dement. 2018;14(2):121-129. doi: 10.1016/j.jalz.2017.10.009

12. Brookmeyer R, Abdalla N. Estimation of lifetime risks of Alzheimer's disease dementia using biomarkers for preclinical disease. Alzheimers Dement. 2018;14(8):981-988. doi: 10.1016/j.jalz.2018.03.005

13. Liu JL, Hlavka JP, Hillestad R, Mattke S. Assessing the preparedness of the U.S. health care system infrastructure for an Alzheimer's treatment. RAND Corporation. Published 2017. Accessed May 10, 2020. rand.org/pubs/research reports/RR2272.html

14. Matthews KA, Xu W, Gaglioti AH, et al. Racial and ethnic estimates of Alzheimer's disease and related dementias in the United States (2015-2060) in adults aged $\geqslant 65$ years. Alzheimers Dement. 2019;15(1):1724. doi: 10.1016/j.jalz.2018.06.3063

15. Hampel H, Caraci F, Cuello AC, et al. A path toward precision medicine for neuroinflammatory mechanisms in Alzheimer's disease. Front Immunol. 2020;11:456. doi: 10.3389/fimmu.2020.00456 16. Johnson EC, Dammer EB, Duong DM, et al. Large-scale proteomic analysis of Azzheimer's disease brain and cerebrospinal fluid reveals early changes in energy metabolism associated with microglia and astrocyte activation. Nat Med. 2020;26(5):769-780. doi: 10.1038/s41591-020-0815-6.

17. An Y, Varma VR, Varma S, et al. Evidence for brain glucose dysregulation in Alzheimer's disease. Alzheimers Dement. 2018;14(3):318-329. doi: 10.1016/j.jalz.2017.09.011

18. Reiman EM, Arboleda-Velasquez JF, Quiroz YT, et al; Alzheimer's Disease Genetics Consortium. Exceptionally low likelihood of Alzheimer's dementia in APOE2 homozygotes from a 5,000-person neuropathological study. Nat Commun. 2020;11(11):667. doi: 10.1038/s41467-019-14279-8 19. Choosing Wisely. American College of Medical Genetics and Genomics. Updated September 15 , 2016. Accessed July 21, 2020. choosingwisely.org/clinician-lists/american-college-medical-geneticsgenomics-apoe-genetic-testing-to-predict-alzheimer-diseasel

20. Selkoe DJ. Light at the end of the amyloid tunnel. Biochemistry. 2018;57(41):5921-5922. doi: $10.1021 /$ acs.biochem. 8 b00985

21. Tolar M, Abushakra S, Sabbagh M. The path forward in Alzheimer's disease therapeutics: reevaluating the amyloid cascade hypothesis. Alzheimers Dement. Published online January 3, 2020. doi: 10.1016/j.jalz.2019.09.075

22. American Geriatrics Society. A guide to dementia diagnosis and treatment. Published 2011. Accessed July 21, 2020. unmfm.pbworks.com/f/American+Geriatric+Society+Dementia+Diagnosis+03-09-11.pdf 23. Vos SJ, Xiong C, Visser PJ, et al. Preclinical Alzheimer's disease and its outcome: a longitudinal cohort study Lancet Neurol. 2013:12(10):957-965. doi: 10.1016/S1474-4422(13)70194-7

24. Amariglio RE, Buckley RF, Mormino EC, et al. Amyloid-associated increases in longitudinal report of subjective cognitive complaints. Alzheimers Dement (N Y). 2018;4:444-449. doi: 10.1016/j.trci.2018.08.005 25. Insel PS. Hansson 0. Mackin RS. Weiner M. Mattsson N: Azheimer's Disease Neuroimaging Initiative. Amyloid pathology in the progression to mild cognitive impairment. Neurobiol Aging. 2018;64:76-84. doi: 10.1016/j.neurobiolaging.2017.12.018

26. Sperling RA, Donohue MC, Raman R, et al; A4 Study Team. Association of factors with elevated amyloid burden in clinically normal older individuals. JAMA Neurol. 2020;77(6):1-11. doi: 10.1001/jamaneurol.2020.0387

27. Ward A, Tardiff S, Dye C. Arrighi HM. Rate of conversion from prodromal Alzheimer's disease to Alzheimer's dementia: a systematic review of the literature. Dement Geriatr Cogn Dis Extra. 2013;3(1):320332. doi: 10.1159/000354370

28. Mitchell AJ, Shiri-Feshki M. Rate of progression of mild cognitive impairment to dementiameta-analysis of 41 robust inception cohort studies. Acta Psychiatr Scand. 2009;119(4):252-265. doi: 10.1111/j.1600-0447.2008.01326.x.

29. Foster NL, Bondi MW, Das R, et al. Quality improvement in neurology: mild cognitive impairment quality measurement set. Neurology. 2019;93(16):705-713. doi: 10.1212/WNL.0000000000008259 30. Owens DK, Davidson KW, Krist AH, et al; US Preventive Services Task Force;: Screening for cognitive impairment in older adults: US Preventive Services Task Force Recommendation Statement. JAMA. 2020;323(8):757-763. doi: 10.1001/jama.2020.0435

31. Fink HA, Hemmy LS, Linskens EJ, et al. Diagnosis and Treatment of Clinical Azheimer's-Type Dementia: A Systematic Review. Agency for Healthcare Research and Quality (US); 2020.

32. Hemmy LS, Linskens EJ, Silverman PC, et al. Brief cognitive tests for distinguishing clinical Alzheimer-type dementia from mild cognitive impairment or normal cognition in older adults with suspected cognitive impairment. Ann Intern Med. 2020;172(10):678-687. doi: 10.7326/M19-3889 33. Johnson KA, Minoshima S. Bohnen NI, et al; Alzheimer's Association: Society of Nuclear Medicine and Molecular Imaging; Amyloid Imaging Taskforce. Appropriate use criteria for amyloid PET: a report of the Amyloid Imaging Task Force, the Society of Nuclear Medicine and Molecular Imaging, and the Alzheimer's Association. Alzheimers Dement. 2013;9(1):e-1-16. doi: 10.1016/j.jalz.2013.01.002

34. Alzheimer's Association. For healthcare professionals: frequently asked questions about beta-amyloid imaging. Published 2020. Accessed May 11, 2020. alz.org/media/Documents/health-care-pros-faqs-betaamyloid-imaging.pdf

35. Rabinovici GD, Gatsonis C, Apgar C, et al. Association of amyloid positron emission tomography with subsequent change in clinical management among Medicare beneficiaries with mild cognitive impairment or dementia. JAMA. 2019;321(13):1286-1294. doi: 10.1001/jama.2019.2000

36. Eli Lilly and Company. Medicines in development. Published April 20, 2020. Accessed May 9, 2020. lilly.com/discovery/clinical-development-pipeline

37. Fleisher AS, Pontecorvo MJ, Devous MD Sr, et al; A16 Study Investigators. Positron emission tomography imaging with [18F]flortaucipir and postmortem assessment of Alzheimer disease neuropathologic changes. JAMA Neurol. Published online April 27, 2020. doi: 10.1001/jamaneurol.2020.0528. 38. Janelidze S. Mattsson N. Palmqvist S, et al. Plasma P-tau181 in Alzheimer's disease: relationship to other biomarkers, differential diagnosis, neuropathology and longitudinal progression to Alzheimer's dementia. Nat Med. 2020;26(3):379-386. doi: 10.1038/s41591-020-0755-1
39. Karikari TK, Pascoal TA, Ashton NJ, et al. Blood phosphorylated tau 181 as a biomarker for Alzheimer's disease: a diagnostic performance and prediction modelling study using data from four prospective cohorts. Lancet Neurol. 2020;19(5):422-433. doi: 10.1016/S1474-4422(20)30071-5 40. Sabbagh MN, Boada M, Borson S, et al. Early detection of mild cognitive impairment (MCI) in primary care. J Prev Alz Dis. 2020;3(7):165-170. doi: 10.14283/jpad.2020.21

41. Wang H, Li T, Barbarino P, et al. Dementia care during COVID-19. Lancet. 2020;395(10231):1190-1191. doi: 10.1016/S0140-6736(20)30755-8

42. Brown EE, Kumar S, Rajji TK, Pollock BG, Mulsant BH. Anticipating and mitigating the impact of the COVID-19 pandemic on Alzheimer's disease and related dementias. Am J Geriatr Psychiatry. 2020;28(7):712-721. doi: 10.1016/j.jagp.2020.04.010

43. Yourish K, Lai R, Ivory D, Smith M. One-third of all U.S. coronavirus deaths are nursing home residents or workers. New York Times. Updated May 11, 2020. Accessed July 21, 2020. nytimes.com/ interactive/2020/05/09/us/coronavirus-cases-nursing-homes-us.html

44. Billioti de Gage S, Moride Y, Ducruet T, et al. Benzodiazepine use and risk of Alzheimer's disease: case-control study. BMJ. 2014;349:05205. doi: 10.1136/bmi..55205

45. Coupland CA, Hill T, Dening T, Morriss R, Moore M, Hippisley-Cox J. Anticholinergic drug exposure and the risk of dementia: a nested case-control study. JAMA Intern Med. 2019;179(8):1084-1093. doi: 10.1001/ jamainternmed.2019.0677

46. Richardson K, Fox C, Maidment I, et al. Anticholinergic drugs and risk of dementia: case-control study. BMJ. 2018;361:k1315. doi: 10.1136/bmj.k1315

47. Scott IA, Anderson K, Freeman CR, Stowasser DA. First do no harm: a real need to deprescribe in older patients. Med J Aust. 2014;201(7):390-392. doi: 10.5694/mja14.00146

48. 2019 American Geriatrics Society Beers Criteria Update Expert Panel. American Geriatrics Society 2019 Updated AGS Beers Criteria for Potentially Inappropriate Medication Use in Older Adults. J Am Geriatr Soc. 2019;67(4):674-694. doi: 10.1111/jgs.15767

49. El-Saifi N, Moyle W, Jones C, Tuffaha H. Medication adherence in older patients with dementia: a systematic literature review. J Pharm Pract. 2018:31(3):322-334. doi: 10.1177/0897190017710524

50. Nguyen TA, Gilmartin-Thomas J, Tan EC, et al. The impact of pharmacist interventions on quality use of medicines, quality of life, and health outcomes in people with dementia and/or cognitive impairment: a systematic review. J Azheimers Dis. 2019:71(1):83-96. doi: 10.3233/JAD-190162

51. Moga DC, Beech BF, Abner EL, et al. INtervention for Cognitive Reserve Enhancement in delaying the onset of Alzheimer's Symptomatic Expression (INCREASE), a randomized controlled trial: rationale, study design, and protocol. Trials. 2019;20(1):806. doi: 10.1186/s13063-019-3993-0

52. Watt JA, Goodarzi Z, Veroniki AA, et al. Comparative efficacy of interventions for aggressive and agitated behaviors in dementia: a systematic review and network meta-analysis. Ann Intern Med. 2019:171(9):633-642. doi: 10.7326/M19-0993

53. Drugs for cognitive loss and dementia. Med Lett Drugs Ther. 2017:59(1530):155-161. 54. Courtney C, Farrell D, Gray R, et al; AD2000 Collaborative Group. Long-term donepezil treatment in 565 patients with Alzheimer's disease (AD2000): randomised double-blind trial. Lancet. 2004;363(9427):2105-2115. doi: 10.1016/S0140-6736(04)16499-4

55. Birks JS, Harvey RJ. Donepezil for dementia due to Alzheimer's disease. Cochrane Database Syst Rev. 2018;6(6):CDO01190. doi: 10.1002/14651858.CDO01190.pub3

56. Rockwood K, Fay S, Gorman M, Carver D, Graham JE. The clinical meaningfulness of ADAS-Cog changes in Alzheimer's disease patients treated with donepezil in an open-label trial. BMC Neurol. 2007:7:26. doi: 10.1186/1471-2377-7-26

57. McShane R. Westby MJ, Roberts E, et al. Memantine for dementia. Cochrane Database Syst Rev. 2019;3(3):CD003154. doi: 10.1002/14651858.CD003154.pub6

58. Choosing Wisely. American Geriatrics Society. Ten things clinicians and patients should question. Updated April 23, 2015. Accessed May 9, 2020. choosingwisely.org/wp-content/uploads/2015/02/AGSChoosing-Wisely-List.pd

59. Niznik JD, Zhao X, He M, et al. Impact of deprescribing AChEls on aggressive behaviors and antipsychotic prescribing. Alzheimers Dement. 2020;16(4):630-640. doi: 10.1002/alz.12054

60. Niznik JD, Zhao X, He M, et al. Risk for health events after deprescribing acetylcholinesterase inhibitors in nursing home residents with severe dementia. J Am Geriatr Soc. 2020;68(4):699-707. doi: 10.1111/jgs.16241 61. Gill SS, Mamdani M. Naglie G, et al. A prescribing cascade involving cholinesterase inhibitors and anticholinergic drugs. Arch Intern Med. 2005;165(7):808-813. doi: 10.1001/archinte.165.7.808 62. Green AR, Segal J, Boyd CM, Huang J, Roth DL. Patterns of potentially inappropriate bladder antimuscarinic use in people with dementia: a retrospective cohort study. Drugs Real World Outcomes. 2020;7(2):151-159. doi: 10.1007/s40801-020-00181-z

63. Bachurin SO, Bovina EV, Ustyugov AA. Drugs in clinical trials for Alzheimer's disease: the major trends. Med Res Rev. 2017;37(5):1186-1225. doi: 10.1002/med.21434

64. Howard R, Zubko O, Bradley R, et al; Minocycline in Alzheimer Disease Efficacy (MADE) Trialist Group. Minocycline at 2 different dosages vs placebo for patients with mild Alzheimer disease: a randomized clinical trial. JAMA Neurol. 2019:77(2):164-174. doi: 10.1001/jamaneurol.2019.3762

65. Ryan J, Storey E, Murray AM, et al; ASPREE Investigator Group. Randomized placebo-controlled trial of the effects of aspirin on dementia and cognitive decline. Neurology. 2020;95(3):e320-e331. doi: 10.1212/WNL.0000000000009277.

66. DeKosky ST, Williamson JD, Fitzpatrick AL, et al; Ginkgo Evaluation of Memory (GEM) Study Investigators. Ginkgo biloba for prevention of dementia: a randomized controlled trial. JAMA. 2008;300(19):2253-2262. doi: 10.1001/jama.2008.683

67. Honig LS, Vellas B, Woodward M, et al. Trial of solanezumab for mild dementia due to Alzheimer's disease. N Engl J Med. 2018;378(4):321-330. doi: 10.1056/NEJMoa1705971

68. Eisai and Biogen to discontinue phase III clinical studies of BACE inhibitor elenbecestat in early Alzheimer's disease. Eisai. News release. Published September 13, 2019. Accessed May 10, 2020. eisai. mediaroom.com/2019-09-13-Eisai-And-Biogen-To-Discontinue-Phase-III-Clinical-Studies-Of-BACEInhibitor-Elenbecestat-In-Early-Alzheimers-Disease

69. Wessels AM, Tariot PN, Zimmer JA, et aL Efficacy and safety of lanabecestat for treatment of early and mild Alzheimer disease: the AMARANTH and DAYBREAK-ALZ randomized clinical trials. JAMA Neurol. 2019;77(2):199-209. doi: 10.1001/jamaneurol.2019.3988 
70. Roche to discontinue phase III CREAD 1 and 2 clinical studies of crenezumab in early Alzheimer's disease (AD) - other company programmes in AD continue. Roche. News release. Published January 30 , 2019. Accessed May 9, 2020. roche.com/dam/jcr:e3181d56-8cac-4db8-a7d4-2f883ee2847c/en/20190130MR_CREN_EN.pdf

71. Egan MF, Kost J, Tariot PN, et al. Randomized trial of verubecestat for mild-to-moderate Alzheimer's disease. N Engl J Med. 2018-378(18):1691-1703 doi: 10.1056/NEJMoa1706441

72. Relkin NR, Thomas RG, Rissman RA, et al; Alzheimer's Disease Cooperative Study. A phase 3 trial of IV immunoglobulin for Alzheimer disease. Neurology. 2017;88(18):1768-1775. doi: 10.1212NNN.0000000000003904 73. Lawlor B, Segurado R, Kennelly S, et al: NILVAD Study Group. Nilvadipine in mild to moderate Alzheimer disease: a randomised controlled trial. PLoS Med. 2018;15(9):e1002660. doi: 10.1371/journal.pmed.1002660 74. Frolich L, Atri A, Ballard C, et al. Open-label, multicenter, phase III extension study of idalopirdine as adjunctive to donepezil for the treatment of mild-moderate Alzheimer's disease. J Alzheimers Dis. 2019:67(1):303-313. doi: 10.3233/JAD-180595

75. Cummings J, Lee G, Ritter A, Sabbagh M, Zhong K. Alzheimer's disease drug development pipeline: 2019. Alzheimers Dement (N Y). 2019:5:272-293. doi: 10.1016/j.trci.2019.05.008

76. Vandenberghe R, Rinne J0, Boada M, et al; Bapineuzumab 3000 and 3001 Clinical Study Investigators. Bapineuzumab for mild to moderate Alzheimer's disease in two global, randomized, phase 3 trials. Alzheimers Res Ther. 2016;8(1):18. doi: 10.1186/s13195-016-0189-7

77. Doody RS, Raman R, Farlow M, et al: Semagacestat Study Group. A phase 3 trial of semagacestat for treatment of Alzheimer's disease. N Engl J Med. 2013;369(4):341-350. doi: 10.1056/NEJMoa1210951 78. US Food and Drug Administration. Early Alzheimer's Disease: Developing Drugs for Treatment. Guidance for Industry. Published February 2018. Accessed May 14, 2020. fda.gov/media/110903/download 79. Morant AV, Jagalski V, Vestergaard HT. Labeling of disease-modifying therapies for neurodegenerative disorders. Front Med (Lausanne). 2019:6:223. doi: 10.3389/fmed.2019.00223

80. Alzforum. CTAD lessons for 2020: more phase 2 trials, more diversity. Clinical trials on Alzheimer's disease 2019. Published January 9, 2020. Accessed May 12, 2020. alzforum.org/news/conferencecoverage/ctad-lessons-2020-more-phase-2-trials-more-diversity

81. Cummings J, Feldman HH, Scheltens P. The "rights" of precision drug development for Alzheimer's disease. Alzheimers Res Ther. 2019;11(1):76. doi: 10.1186/s13195-019-0529-5

82. Cole MA, Seabrook GR. On the horizon-the value and promise of the global pipeline of Alzheimer's disease therapeutics. Alzheimers Dement (N Y). 2020;6(1):e12009. doi: 10.1002/trc2.12009

83. Alzforum. Aducanumab. Updated July 17, 2020. Accessed July 21, 2020. alzforum.org/ therapeutics/aducanumab

84. Schneider L. A resurrection of aducanumab for Alzheimer's disease Lancet Neurol 2020:19(2):111112. doi: $10.1016 / S 1474-4422(19) 30480-6$

85. Biogen. Aducanumab update. Updated October 28, 2019. Accessed May 12, 2020. investors.biogen. $\mathrm{com} /$ static-files/5a31a1e3-4fbb-4165-921a-f0ccb1d64b65

86. Haeberlein SB, von Hehn C, Tian Y, et al. EMERGE and ENGAGE topline results: two phase 3 studies to evaluate aducanumab in patients with early Alzheimer's disease. CTAD 2019. December 19, 2019. Accessed May 12, 2020. investors.biogen.com/static-files/ddd45672-9c7e-4c99-8a06-3b557697c06f 87. Alzforum. "Reports of My Death Are Greatly Exaggerated." Signed, Aducanumab. Published October 24, 2019. Accessed May 12, 2020. alzforum.org/news/research-news/reports-my-death-are-greatlyexaggerated-signed-aducanumab

88. Biogen. Biogen reports Q1 2020 revenues of $\$ 3.5$ billion. Published April 22, 2020. Accessed May 12 2020. investors.biogen.com/static-files/60eda571-f103-42ee-8b03-5c64571dfc13

89. Biogen. Biogen completes submission of Biologics License Application to FDA for aducanumab as a treatment for Alzheimer's disease. Published July 8, 2020. Accessed July 15, 2020. investors.biogen.com/ news-releases/news-release-details/biogen-completes-submission-biologics-license-application-fda 90. A Study to Confirm Safety and Efficacy of BAN2401 in Participants With Early Alzheimer's Disease (Clarity AD). Updated March 16, 2020. Accessed May 12, 2020. clinicaltrials.gov/ct2/show/NCT03887455 91. Alzheimer's Clinical Trials Consortium and Eisai. Alzheimer's Clinical Trials Consortium selects elenbecestat and BAN2401 for upcoming clinical studies on prevention of Alzheimer's disease. Published May 10, 2019. Accessed May 9, 2020. eisai.com/news/2019/pdf/enews201932pdf.pdf?fbclid=IwAR20unfoUw19WxiSIQJv3KR5tgfohjNyPA97bkXCMPEX51enLe1KyLV-6s

92. Alzheimer's Clinical Trials Consortium. AHEAD 3-45 Study. 2019. Accessed June 10, 2020. a3a45.org 93. Roche provides topline results from investigator-led phase I//III trial with gantenerumab in rare inherited form of Alzheimer's disease. Roche. News release. February 10, 2020. Accessed May 9, 2020. roche. com/media/releases/med-cor-2020-02-10.htm
94. Gauthier S, Aisen PS, Cummings J, et al; EU/US CTAD Task Force. Non-amyloid approaches to disease dodification for Alzheimer's disease: an EU/US CTAD Task Force report. J Prev Alzheimers Dis. doi: 10.14283/jpad.2020.182020:1-6

95. Wilcock GK, Gauthier S, Frisoni GB, et al. Potential of low dose leuco-methylthioninium bis(hydromethanesulphonate) (LMTM) monotherapy for treatment of mild Alzheimer's disease: cohort analysis as modified primary outcome in a phase III clinical trial. J Alzheimers Dis. 2018;61(1):435-457. doi: 10.3233/JAD-170560

96. Safety and Efficacy of TRx0237 in Subjects With Alzheimer's Disease Followed by Open-Label Treatment. Updated July 15, 2020. Accessed July 21, 2020. clinicaltrials.gov/ct2/show/NCT03446001 97. Safety and Efficacy Study of ALZT-OP1 in Subjects With Evidence of Early Alzheimer's Disease (COGNITE). Updated May 1, 2020. Accessed May 12, 2020. clinicaltrials.gov/ct2/show/NCTO2547818 98. Detke M. Lynch C. Holsinger LJ, et al. Poster P4-663: Initiation of the phase 2/3 GAIN trial of COR388, a novel bacterial virulence factor inhibitor for the treatment of Alzheimer's disease based on phase $1 \mathrm{a} / \mathrm{b}$ safety, PK, biomarker, and efficacy data. Alzheimers Dement. 2019;15(7 suppl):P1585.

99. Cortexyme provides regulatory update for COR388 development program in Alzheimer's disease. BioSpace. Published February 13, 2020. Accessed May 6, 2020. biospace.com/article/cortexyme-providesregulatory-update-for-cor388-development-program-in-alzheimer-s-disease/

100. AB Science. Interim results of masitinib study in Alzheimer's disease: positive trend of efficacy in one of the doses tested. Published June 26, 2019. Accessed May 12, 2020. ab-science.com/images/_pdf/ CP_AD_Interim_VEng_VF.pdf

101. Masitinib in Patients With Mild to Moderate Alzheimer's Disease. Updated April 7, 2020. Accessed May 12, 2020. clinicaltrials.gov/ct2/show/NCT01872598

102. Study of AGB101 in Mild Cognitive Impairment Due to Alzheimer's Disease (HOPE4MCI). Updated April 3, 2020. Accessed May 12, 2020. clinicaltrials.gov/ct2/show/NCT03486938

103. Hampel $H$, Williams C, Etcheto $A$, et al. A precision medicine framework using artificial intelligence for the identification and confirmation of genomic biomarkers of response to an Alzheimer's disease therapy: analysis of the blarcamesine (ANAVEX2-73) phase 2a clinical study. Alzheimers Dement (N Y). 2020:6(1):e12013. doi: 10.1002/trc2.12013

104. OLE for Patients Enrolled in Phase 2b/3 Study ANAVEX2-73-AD-004 (ATTENTION-AD). Updated March 19, 2020. Accessed May 12, 2020. clinicaltrials.gov/ct2/show/NCT04314934

105. Vandenberghe R, Riviere ME, Caputo A et al. Active AB immunotherapy CAD106 in Alzheimer's disease: a phase 2b study. Alzheimers Dement (N Y). 2017;3(1):10-22. doi: 10.1016/j.trci.2016.12.003 106. Lopez Lopez C, Tariot PN, Caputo A, et al. The Alzheimer's Prevention Initiative Generation Program: study design of two randomized controlled trials for individuals at risk for clinical onset of Alzheimer's disease. Alzheimers Dement (N Y). 2019:5:216-227. doi: 10.1016/j.trci.2019.02.005

107. A Study of CAD106 and CNP520 Versus Placebo in Participants at Risk for the Onset of Clinical Symptoms of Alzheimer's Disease (Generation S1). Updated June 18, 2020. Accessed July 21, 2020. clinicaltrials.gov/ct2/show/NCTO2565511

108. Brain Amyloid and Vascular Effects of Eicosapentaenoic Acid (BRAVE-EPA). Updated April 15, 2020. Accessed May 12, 2020. clinicaltrials.gov/ct2/show/NCT02719327

109. Boada M, López O, Núñez L, et al. Plasma exchange for Alzheimer's disease Management by Albumin Replacement (AMBAR) trial: study design and progress. Alzheimers Dement (N Y). 2019;5:61-69. doi: 10.1016/j.trci.2019.01.001

110. Grifols presents its latest Alzheimer's clinical trial data. Grifols. News release. Published December 6. 2019. Accessed May 12, 2020. intranet.grifols.com/documents/38081080/1022159504/np-20191206-en. $\mathrm{pdf} / \mathrm{a} 779 \mathrm{e} 23 \mathrm{c}-2 \mathrm{c} 8 \mathrm{a}-4 \mathrm{~d} 5 \mathrm{a}-915 \mathrm{e}-\mathrm{f} 3536 \mathrm{f} 6 \mathrm{e}-5 \mathrm{~b} 6 \mathrm{e}$

111. Imbimbo BP, Ippati S, Ceravolo F, Watling M. Perspective: is therapeutic plasma exchange a viable option for treating Alzheimer's disease? Alzheimers Dement (N Y). 2020;6(1):e12004. doi: 10.1002/ trc2.12004

112. Study of BHV-4157 in Alzheimer's Disease (T2 Protect AD). Updated February 17, 2020. Accessed May 12, 2020. clinicaltrials.gov/ct2/show/NCT03605667

113. Carlisle BG. Clinical trials stopped by Covid-19. Published May 5, 2020. Accessed May 9, 2020. covid19.bgcarlisle.com/published/full-report-2020-05-07.html

114. Alzforum. Coronavirus takes its toll on Alzheimer's clinical studies. Published March 27, 2020 Accessed May 9, 2020. alzforum.org/news/community-news/coronavirus-takes-its-toll-alzheimersclinical-studies

115. Langa KM, Burke JF. Preclinical Alzheimer disease-early diagnosis or overdiagnosis? JAMA Intern Med. 2019;10.1001/jamainternmed.2019.2629. doi: 10.1001/jamainternmed.2019.2629 


\title{
Economic Burden of Alzheimer Disease and Managed Care Considerations
}

\author{
Winston Wong, PharmD
}

\section{Introduction}

Dementia is a term for any disease causing a change in memory that impairs a person's daily functioning. ${ }^{1}$ Alzheimer disease (AD), the most common type of dementia, is a degenerative disease characterized by loss of memory, loss of cognitive function, and functional impairment, with associated neuropsychological symptoms. As $\mathrm{AD}$ is a progressive disease, patients can be classified as having preclinical $\mathrm{AD}$, mild cognitive impairment (MCI) due to $\mathrm{AD}$, and mild, moderate, or severe dementia due to $\mathrm{AD}$ along the disease continuum. MCI is defined as deficits in memory with no significant impact on daily functioning, whereas MCI due to AD is defined as evidence of $\mathrm{AD}$ pathology and impairment in one or more cognitive domains that does not interfere with daily functioning. MCI can be a result of several conditions including, but not limited to, $\mathrm{AD}$, cerebrovascular disease, Parkinson disease, frontotemporal degeneration, or traumatic brain injury. However, patients with $\mathrm{MCI}$ are more likely to develop AD than those who do not have MCI, suggesting that MCI may be an early sign of AD. ${ }^{2-7}$ Evaluation of patients with MCI and other risk factors for AD may help in early identification and diagnosis of AD.

$\mathrm{AD}$ has been identified as the sixth leading cause of death among adults in the United States, and the fifth leading cause of death among adults over 65 years. ${ }^{8}$ In 2018 , more than 122,000 people died from $\mathrm{AD}$, an increase of $146 \%$ from the year 2000 . An estimated 5.8 million adults over 65 years are living with $\mathrm{AD}$, with the number expected to more than double by the year 2050 to approximately 14 million individuals. A diagnosis of AD is typically not made until the patient has progressed to mild or moderate dementia. Many patients with AD go undiagnosed, and about $50 \%$ of Medicare patients are unaware they have a documented diagnosis of AD.

When evaluating the economic burden of $\mathrm{AD}$, it is important to consider direct costs, such as skilled nursing care, home healthcare, and long-term care, and indirect costs, such as quality of life and the impact on the caregiver. Indirect costs of care have been shown to be higher than the costs of direct care for patients with $\mathrm{AD}$ and should not be overlooked when evaluating the cost of care for patients with AD. ${ }^{9}$ Many patients living with $\mathrm{AD}$ are undiagnosed;

\section{ABSTRACT}

Alzheimer disease is the most common cause of dementia and the fifth leading cause of death in adults older than 65 years. The estimated total healthcare costs for the treatment of Alzheimer disease in 2020 is estimated at $\$ 305$ billion, with the cost expected to increase to more than $\$ 1$ trillion as the population ages. Most of the direct costs of care for Alzheimer disease are attributed to skilled nursing care, home healthcare, and hospice care. Indirect costs of care, including quality of life and informal caregiving, are likely underestimated and are associated with significant negative societal and personal burden. Managed care organizations are in a unique position to develop utilization strategies that would positively impact early diagnosis and treatment to lead to better outcomes and lower costs for patients, caregivers, and the healthcare system. Additionally, the recent inclusion of Alzheimer disease diagnoses into risk corridor calculations by the Centers for Medicare \& Medicaid Services may encourage Medicare Advantage organizations to invest in programs that aid in its early detection and diagnosis.

Am J Manag Care. 2020;26:S177-S183

For author information and disclosures, see end of text. 
therefore, estimating true costs of AD care is difficult. The incorporation of medication therapy management (MTM) into AD care is an important strategy to optimize early medication initiation. Early detection and diagnosis of $\mathrm{AD}$, leading to early initiation of current AD therapies, is associated with improved quality of care and quality of life, and economic and caregiver outcomes. Barriers to early diagnosis of AD include healthcare provider time restraints regarding testing and counseling, hesitancy of patient and caregiver reporting of symptoms, and lack of diagnostic resources in primary care. ${ }^{3-6,10,11}$

\section{Direct and Indirect Costs of AD}

As the prevalence of $\mathrm{AD}$ continues to rise with the aging "baby boomer" population, so do the costs of care associated with $\mathrm{AD}$. The total cost of care for the treatment of AD in 2020 is estimated to be $\$ 305$ billion. Medicare and Medicaid cover the largest proportion of these costs, estimated to be around $\$ 206$ billion ( $\approx 68 \%$ ), while patient out-of-pocket (OOP) costs are estimated to be around $\$ 66$ billion $(\approx 22 \%)^{2,12}\left(\right.$ Table $\left.^{2}\right)$.

Total costs for $\mathrm{AD}$ care are estimated to increase to more than $\$ 1$ trillion by 2050 . Direct medical costs associated with the treatment of AD include physician visits, emergency department and hospital admissions, long-term care or skilled nursing facility care, and medications. Direct nonmedical costs include home healthcare, transportation to medical visits, and modifications to adapt to changes in physical function. Long-term care and nursing home care costs account for the majority of direct costs associated with AD care. ${ }^{13}$

Direct costs of care reported for AD may vary depending on the time frame being evaluated, and may include analysis of end-of-life costs, lifetime costs, pre- and postdiagnosis costs, and prevalencebased costs. ${ }^{13}$ Total Medicare costs in 2019 for patients 65 years and older with $\mathrm{AD}$ or other dementias have been estimated to be $\$ 25,213$ per person, about 3 times higher than those without AD (\$7750). Medicaid pays for nursing home and long-term care services for patients with low income who meet certain criteria. The average annual Medicaid payments for beneficiaries with AD are estimated to be 23 times higher than those without AD (average $\$ 8779$ vs $\$ 374$ ). ${ }^{2}$

TABLE. Estimated Total Cost of Care for the Treatment of Alzheimer Disease in 2020

Payment Source Cost in Billions (B)

\begin{tabular}{lc} 
Medicare & $\$ 155 \mathrm{~B}$ \\
Medicaid & $\$ 51 \mathrm{~B}$ \\
\hline Out of pocket & $\$ 66 \mathrm{~B}$ \\
\hline Other & $\$ 33 \mathrm{~B}$ \\
\hline Total cost & $\$ 305 \mathrm{~B}$ \\
\hline
\end{tabular}

Three studies evaluated the direct costs of care for patients with $\mathrm{AD}$ around the time of death. A retrospective analysis evaluated 338,288 beneficiaries older than 69 years. Of these beneficiaries, $21 \%$ were classified as having AD. Beneficiaries with AD had costs on average of at least $\$ 18,000$ more than those who did not have $A D$ in the 8 years before death plus the year of death $(P<.01)$, with most of these costs associated with skilled nursing care, home healthcare, and hospice care as compared with intensive medical treatments such as Medicare Part B drugs $(P<.01)$. Additionally, patients with $\mathrm{AD}$ had used $11 \%$ higher costs over the last 8 years of life compared to those who did not have AD. ${ }^{14}$ Kelley et al showed the mean adjusted total healthcare spending in the last 5 years of life was $\$ 287,038$ in patients with dementia, compared with $\$ 183,001$ in other disease groups (eg, cancer, heart disease).$^{15}$ Another study estimated the cost of dementia to Medicare and Medicaid by analyzing the 1997 to 2005 Medicare Current Beneficiary Survey. Using cohort-based simulation models, the costs of dementia were estimated from the time of diagnosis until death. Patients with dementia had significantly higher annual Medicare and Medicaid expenditures at $\$ 10,814$ and $\$ 6234$ compared with those without dementia at $\$ 5953$ and $\$ 1962$, respectively $(P<.05) .{ }^{16}$

One study evaluated direct costs of care for patients with $\mathrm{AD}$ around the time of diagnosis. A retrospective, observational cohort study using a 5\% sample of the 2009 to 2013 Medicare claims files evaluated Medicare expenditures during the 24 months before and after the diagnosis of AD and related dementia (ADRD) or MCI, each with propensity score-matched controls. Patients in the ADRD group were older and had more comorbidities when compared with those in the MCI or control groups. During months 13 to 24 before diagnosis, the average Medicare costs for patients in the ADRD group were $\$ 10,533$. Costs increased to an average of $\$ 15,091$ in the 12 months before diagnosis. The 12 months after diagnosis were found to be the most costly, totaling on average $\$ 27,126$, and decreased during months 13 to 24 after diagnosis to an average of $\$ 17,257$ due to a decrease in inpatient and acute care. Utilization of inpatient care, home healthcare, and post-acute skilled nursing facility care significantly increased in the 12 months before and after diagnosis. Patients in the MCI group experienced similar trends as patients in the ADRD group when compared with the control group. ${ }^{17}$

As skilled nursing care, home healthcare, and hospice care have been identified as major drivers of direct costs of care in $\mathrm{AD}$, it is important to review healthcare utilization in these settings. It is estimated that $32 \%$ of patients using home healthcare services, $42 \%$ of patients in residential care facilities, and $48 \%$ of nursing home residents have AD. ${ }^{2}$ Goldfeld and colleagues evaluated 323 nursing home residents over 18 months with advanced dementia in a prospective, cohort study to examine the factors associated with increased Medicare costs. The average total Medicare expense over 18 months was $\$ 8522$ per resident. Medicare expenditures 
were highest for hospice care (45.6\%), followed by hospitalizations (30.2\%). Medicare spending was found to be highest in the last 90 days before death. ${ }^{18}$ Additionally, OOP costs for patients with AD are estimated to be higher than OOP costs for patients without AD. A retrospective, cross-sectional study using data from the 2012 Medicare Current Beneficiary Study aimed to estimate the OOP healthcare spending associated with ADRD. The average annual per-capita OOP spending was $\$ 3285$ in patients with ADRD and $\$ 1895$ in patients without ADRD. The majority of OOP spending in patients with ADRD was for prescription drugs and home healthcare services. ${ }^{19}$

Indirect costs of care associated with $\mathrm{AD}$ include caregiver burden and associated healthcare utilization and costs. ${ }^{13}$ Family and friends may take on the burden of caregiving due to a sense of obligation and love, and desire to keep their loved ones comfortable in their own homes. This desire to keep loved ones at home is supported by the observation that patients with $\mathrm{AD}$ and dementia will be less agitated and more comfortable in surroundings that they recognize and are familiar with. Informal caregiving is the unpaid care that is provided to a patient with AD by their family and friends. It has been estimated that $75 \%$ of caregiving for a patient with $\mathrm{AD}$ is informal care. ${ }^{13}$ Providing care to a patient with AD is financially, physically, and emotionally burdensome to the caregiver. Caregiving for patients with $\mathrm{AD}$ is unique compared with caregiving in other disease states due to the long duration of disease, and the progressive, unrelenting decline in cognitive and physical functioning. ${ }^{2}$ The majority of $\mathrm{AD}$ care consists of assisting with activities of daily living (ADLs), but many more AD caregivers report also handling finances and advocating in terms of healthcare than non-AD caregivers. Other caregiver tasks for patients with $\mathrm{AD}$ include ensuring medication compliance, managing behavioral symptoms of the disease, finding and participating in support services such as adult day programs, and organizing in-home care. . $20^{20}$

Several studies have examined the impact of AD on the caregiver. When evaluating the financial impact of informal caregiving in $\mathrm{AD}$, researchers have typically expressed these costs as either replacement cost of hiring formal care or forgone wages. In 2019, 16.3 million informal caregivers provided an estimated 18.6 billion hours of unpaid care. When evaluating the cost using replacement costs of care valued at $\$ 13.11$ per hour, the estimated value of informal care provided in 2019 was $\$ 244$ billion. $^{2}$ A retrospective cohort study evaluated social costs and financial risks in Medicare fee-for-service beneficiaries older than 70 years from the Health and Retirement Study in the 5 years before their death between 2010 and 2015. This study found that costs of informal care for patients with AD were significantly higher than those with other conditions, including cancer and heart disease. The costs of informal care, measured in this study by replacement costs, were found to be $\$ 83,022$ for patients with AD versus $\$ 38,272$ for other diseases. ${ }^{15}$
A large, multicenter, prospective cohort study used bivariate probit models to estimate home health and informal care utilization and costs in patients with AD from the Predictors Study over a span of 7 years. This study estimated informal caregiving costs by using the national average hourly earning for all private industries for each year. The majority of patients received informal care (80.6\%), which increased from 4 hours of care per day at baseline to 7.6 hours per day at year 4 . The costs of informal care were estimated to be $\$ 20,590$ for the baseline year, and increased to $\$ 43,031$ in year $4 .{ }^{21}$ It is estimated that informal caregiver costs increase $18 \%$ per year as symptoms of AD progress. ${ }^{22}$ Lastly, one study estimated the "welfare cost," a more comprehensive cost estimate of informal caregiving that takes into account the value of time, implications for future employability, and intrinsic benefits that accrue to daughters caring for mothers with $\mathrm{AD}$, and the cost of forgone wages in the same population. When comparing cost estimates of informal caregiving over 2 years, the average cost in forgone wages was estimated to be $\$ 24,500$ over all health states (needing assistance with ADLs only, memory only, both, or cannot be left alone) compared with welfare cost of $\$ 180,000$. This study highlights that the financial impact on the informal caregiver may be much greater than other current estimates, and estimating by forgone wage or replacement costs, provide underestimates of informal care costs. ${ }^{23}$

The impact on physical health of the caregiver and the associated healthcare utilization and costs are often overlooked. Caregivers to patients with $\mathrm{AD}$ have been shown to have higher rates of stress, depression, anxiety, physical ailments, increased cardiovascular disease, and weakened immune systems. One study showed that as the comorbid diseases in patients with $\mathrm{AD}$ progressed and dependence on the caregiver increased, healthcare utilization and costs of the caregiver increased. ${ }^{24}$ A retrospective cohort study using Medicare Advantage Prescription Drug Plan members with a diagnosis of $\mathrm{AD}$ and their household members compared with those without $\mathrm{AD}$ evaluated the medical condition burden, healthcare usage, and healthcare costs of the caregiver. Rheumatoid arthritis, mood and anxiety disorders, insomnia, and substance use/abuse were significantly more prevalent in household members caring for patients with AD. Household members caring for patients with AD had significantly higher average annual healthcare costs ( $\$ 7168$ $\pm \$ 10,050$ vs $\$ 6301 \pm \$ 8311 ; P<.001) .{ }^{25}$ Therefore, it is imperative to support and treat the caregiver as much as the patient. Caregiver interventions include case management, educational and psychotherapeutic approaches, respite care options, and support groups. Education on the progression of the disease, how to find and access help, and optimal usage of resources to assist in providing AD care have shown to have a positive impact on AD caregivers.

The quality of life (QOL) in patients with $\mathrm{AD}$ is also significantly negatively impacted. Dementia interferes with daily functioning and independence, and places not only an economic burden, but also 
a heavy personal burden on the patient and their caregivers. The symptoms of dementia are disabling, the degree to which depend largely on the stage of AD. Symptoms include loss of memory, difficulty speaking, psychological and psychiatric changes, and the inability to perform ADLs. ${ }^{2}$ According to Lawton's model of QOL in $\mathrm{AD}$, physiological well-being, behavioral competence (eg, cognitive and functional ability), and objective environment (eg, caretakers and living situation) are the 3 main factors that contribute to QOL in patients with $\mathrm{AD} .{ }^{26}$ The long duration of $\mathrm{AD}$ contributes to the negative impact it has on public health and decreases in QOL. To quantify this, disability-adjusted life-years (DALYs) assess the number of years of life lost due to a disease. DALYs are calculated as the number of years of life lost (YLLs) due to premature mortality plus the number of years lived with disability (YLDs), totaled across all those with the disease or injury. ${ }^{2}$ According to The State of US Health, 1990-2016: Burden of Diseases, Injuries, and Risk Factors Among US States, AD ranked number 4 in YLLs and 19 in YLDs, indicating the high burden of disease to the patient and the caregiver. ${ }^{27}$ Although it is known that QOL is negatively impacted in individuals with $\mathrm{AD}$, there is a paucity in data quantifying the cost of decreased QOL in the patient.

\section{Early Treatment Optimization Strategies}

The overall goals of treatment for patients with $\mathrm{AD}$ are to maintain QOL; maximize function in daily activities; enhance cognition, mood, and behavior; foster a safe environment; and promote social engagement. These goals are achieved by regular monitoring of health and cognition in patients with $\mathrm{AD}$, providing education and support to patients and their caregivers, and initiation of pharmacologic and nonpharmacologic interventions. ${ }^{28}$ The National Plan to Address Alzheimer's Disease was established in 2012 to fulfill the 2011 National Alzheimer's Project Act (NAPA), which established a set of goals to address the current needs of patients with AD and prevent future cases of AD and ADRD. These goals include prevention and effective treatment of AD by 2025 , optimizing care quality and efficiency, expanding support for patients with AD and their families and caregivers, enhancing public awareness and engagement, and tracking progress to drive improvement. ${ }^{29}$

Unfortunately, most patients with AD are diagnosed in the late stages of the disease when the symptoms are noticeable, with very few patients being diagnosed in the preclinical stages. As a response to the detrimental impacts and costs of misdiagnosed or undiagnosed $\mathrm{AD}$, new practice guidelines for the clinical evaluation of $\mathrm{AD}$ in primary and specialty care settings were released at the 2018 Alzheimer's Association International Conference. These guidelines sought to improve and coordinate efforts for more timely and accurate AD diagnosis and evaluation of symptoms, as well as providing continued care and support for the affected individual and their caregivers. The guidelines focus on recommendations for appropriate, timely evaluation and assessment, and highlight the importance of including the caregiver in all aspects of AD care. ${ }^{30}$

In its recent practice recommendations, the Alzheimer's Association renewed the focus of care on the individual with dementia and their caregiver in a person-centered care delivery model that focuses more on the individual's unique needs, personal experiences, and strengths rather than the loss of abilities. ${ }^{2}$ The Alzheimer Association guidelines for the treatment of dementiarelated behaviors indicate that nonpharmacologic psychosocial interventions should be used as first-line interventions. For the treatment of cognitive symptoms of $\mathrm{AD}$, current pharmacologic agents approved for use in the treatment of patients with $\mathrm{AD}$ at various stages of the disease include cholinesterase inhibitors (donepezil, galantamine, and rivastigmine) and memantine. ${ }^{31}$ These current pharmacologic therapies for AD provide symptomatic relief by improving cognitive symptoms of memory loss and confusion, but do not stop AD progression. ${ }^{32}$ Promising disease-modifying therapies that target inflammatory processes, tau proteins, and $\beta$-amyloid proteins are currently in development.

In the preclinical stages of $\mathrm{AD}$, patients do not show signs of MCI or dementia, but may have measurable changes in the brain that can be detected on positron emission tomography scans, by specific biomarkers, possibly by retinal nerve fiber layer thickness as detected on optical coherence tomography, or other changes that may be detected through varying laboratory and genetic tests that are in development. ${ }^{2,33}$ Patients with MCI are more likely to have $\mathrm{AD}$ than those who do not have MCI, suggesting that MCI may be an early sign of AD. If MCI is properly detected and diagnosed, this may lead to an earlier diagnosis of AD..$^{34,35}$

Early diagnosis of AD can lead to earlier interventions, both pharmacologic and nonpharmacologic, to help maintain and improve physical and cognitive functioning. ${ }^{36}$ Early diagnosis may lead to earlier interventions, such as coordinated care planning involving the affected individual, better management of symptoms, reduced costs, and better QOL for the patient and caregiver. ${ }^{37}$ One study evaluated the costs and benefits of early identification and treatment of patients with $\mathrm{AD}$ using a Monte Carlo cost-benefit analysis. This analysis showed that early diagnosis and management of patients with AD with pharmacologic and nonpharmacologic measures would lead to cost savings, with cost savings being greatest when AD cases were diagnosed earlier. Cost savings were greatest when drug treatment in combination with caregiver interventions (eg, increased counseling and support) were initiated when cognitive impairment was lower. For example, a Monte Carlo cost-benefit analysis predicted that the net savings for a 70-year-old woman with MCI (defined as a Mini-Mental State Examination score of 28) may be estimated at $\$ 125,000$ in social benefits, $\$ 16,000$ in state fiscal benefits, and $\$ 34,000$ in federal fiscal benefits. ${ }^{38}$ De Vugt and colleagues argue that early diagnosis of $\mathrm{AD}$ allows the informal 
caregiver to have more time to adjust to their role in providing care for their loved one with $\mathrm{AD}$, but highlight the importance of the caregiver finding the support they need in caring for a patient with AD. While stress levels may be lower in the preclinical stages of AD, early interventions and support can help the caregiver anticipate and plan for future care needs and allow the patient to be involved in the decision-making process. ${ }^{39}$ More studies assessing the impact of early diagnosis and early intervention on quality of life, caregiver burden, and economic burden are needed as more patients receive a diagnosis earlier.

Managed care organizations (MCOs) have a unique opportunity in developing medication utilization strategies that support the goals of the National Plan and have an impact on early diagnosis and treatment that can lead to better outcomes and lower costs for patients, caregivers, and the healthcare system. While there is promise in the various emerging treatment options to modify the progression of $\mathrm{AD}$, the current pharmacologic therapies that provide temporary, symptomatic relief are associated with significant adverse effects that often lead to noncompliance. Appropriate utilization of current pharmacotherapy options and care coordination is important for managing costs of $\mathrm{AD}$, as well as optimizing QOL and care for patients with AD and their caregivers.

To achieve this, MCOs must first prioritize and encourage appropriate, timely testing for cognitive status earlier in the disease process. MCOs can prioritize review of emerging treatment options that target inflammatory processes, tau proteins, and $\beta$-amyloid proteins as they become available. One study suggests that the maximum effective price of these potential disease-modifying therapies per patient per year is $\$ 10,000$ from a payer perspective under the willingness-to-pay threshold of $\$ 150,000$ per qualityadjusted life-year, assuming 20\% reduction relative to standard of care. ${ }^{40}$ Care planning should be initiated for all patients with $\mathrm{AD}$, as it provides individuals with cognitive impairment and their caregivers information on medical and nonmedical treatments, and helps to coordinate care among clinicians vital to the care of patients with $\mathrm{AD}{ }^{41}$

MCOs can also use MTM approaches to help initiate and maintain patients on currently approved pharmacologic agents in the treatment of AD early in the disease process. MTM is a process of collecting patient-specific information, assessing medication therapies to identify medication-related issues, and creating a plan to resolve these issues. The core elements of MTM services include medication therapy review, personal medication record, medication-related action planning, intervention/referral, and documentation and follow-up. MTM is especially important in chronic disease states with several comorbidities. Disease management programs that incorporate MTM have been developed for $\mathrm{AD}$, as patients with $\mathrm{AD}$ and their caregivers manage this complex, progressive disease and associated comorbidities at home. Disease management programs that incorporate MTM services have been shown to reduce healthcare costs and improve QOL of the patient and caregiver. ${ }^{42}$ Additionally, caregiver coaching can help to educate, prepare, and train the caregiver to minimize caregiver burden. Caregiver coaching includes, but is not limited to, education on $\mathrm{AD}$, how to manage changes in behavior and communication, personal care and hygiene tips, home safety and fall prevention strategies, medication management, how to manage financial and legal matters, and emergency procedures. There are many resources and support groups available for caregivers of patients with $\mathrm{AD}$, and it is imperative for the caregiver to have a support team while caring for their loved one with AD. ${ }^{43}$

The Centers for Medicare \& Medicaid Services (CMS) uses a risk-adjustment mechanism, currently the Hierarchical Condition Category (HCC) risk adjuster, to calculate revenue payments made to Medicare Advantage organizations (MAOs) for their Part C Medicare Advantage (MA) plans. This is done so that MAOs do not select their members by their risk score, but allows for a market where quality and efficiency of service is optimized. Risk scores are calculated by patient medical diagnoses submitted to CMS from the prior year, which are then used to calculate member-specific revenue payments, which make up most of the revenue of the plan. Thorough diagnosis coding and submission, and retention of members, directly impacts the ability of the MAO to maintain and increase their revenue. ${ }^{44} \mathrm{CMS}$ released its proposed risk score methodology for MA plans for 2020 in December 2018. In January 2020, CMS included 2 additional HCC risk adjusters for patients with dementia, which includes patients with $\mathrm{AD}$ to the risk score calculation methodology. Therefore, it is in the MAO's best interest to actively identify and track costs for patients with $\mathrm{AD}$ and dementia to be included in their risk-adjustment calculations. MAOs are encouraged to provide care to patients with $\mathrm{AD}$ and dementia because the costs are already included in their risk-adjustment calculations and are being paid to provide the services. ${ }^{45}$ This will also encourage MAOs to invest in programs that aid in early detection and diagnosis of AD.

In addition to pharmacologic therapy, early nonpharmacologic interventions, such as learning basic strengthening exercises and learning how to use basic self-help assistive devices while patients with AD have the capacity to learn them, can help patients maintain autonomy in ADLs and physical and cognitive functioning. The American Academy of Neurology recommends that patients with MCI exercise regularly as part of an overall approach to managing their symptoms and to consider cognitive training. ${ }^{46}$ Physical and occupational therapy for learning and maintaining fine and gross motor function skills are important aspects of nonpharmacologic management to help maintain ADLs. Physical therapists are well trained to assist in exercising by using specific methods, techniques, and approaches to help maintain physical functioning in patients with $\mathrm{AD}$, including sight, sound, and touch cues for 
walking, mirroring movements, task breakdown to safely get out of bed, hand-over-hand guidance to learn different motions, and muscle training to help patients walk safely. ${ }^{47}$ Occupational therapists can evaluate the home for safety to prevent falls or injuries, create safety plans to keep the patient safe while at home when the caregiver is unavailable, and observe the patient at home to recommend changes to foster independence. ${ }^{48}$ Various cognitive training programs, including cognitive stimulation therapy (CST), are being considered for coverage by MA plans under supplement benefit funds from CMS. ${ }^{49,50} \mathrm{CST}$ has been shown to be a cost-effective early intervention in patients with dementia, and is typically delivered in a group setting consisting of cognitive-based tasks and activities, such as word games and puzzles. A multicenter, single-blind, randomized control trial evaluated the effect of CST on cognition using the AD assessment scale-cognition (ADAS-Cog) in patients with AD. CST showed a significant impact in improving the ability to follow commands and spoken language ability in patients with $\mathrm{AD}(P<.05) .{ }^{51}$ The use of assistive devices for ADLs can help patients with AD maintain their independence, and includes devices such as button, zipper, and sock aids to help get dressed, and modified utensils, plates, and bowls to assist in eating. ${ }^{52,53}$ Technological advances in medical equipment for patients with dementia who have several comorbidities with high pill burden, such as automated pill dispensers that remind patients with $\mathrm{AD}$ to take their medications, may have a positive impact on medication management and reduce healthcare utilization for comorbid disease states. MCOs should consider coverage of nonpharmacologic therapies and tools that have shown to be cost-effective, and increase QOL in patients with $\mathrm{AD}$ and their caregivers.

\section{Conclusions}

$\mathrm{AD}$ is prevalent among adults older than 65 years and is often misdiagnosed or underdiagnosed. The economic burden from treating patients with $\mathrm{AD}$ is overwhelming, and estimated to increase in the coming years as the population ages. The direct costs of care for patients with $\mathrm{AD}$ are largely attributed to skilled nursing care, home healthcare, and hospice care, and have been shown to be significantly more costly for patients with $\mathrm{AD}$ as compared with other diseases. The costs of informal care and QOL burden have also been shown to be significantly higher in patients with AD and their caregivers than other disease states. The financial and personal burden experienced by caregivers to patients with AD is immense and often underestimated. It is important to treat and support the caregiver of patients with AD just as much as the patient with AD. MCOs can have a profound impact on the QOL of the patient with $\mathrm{AD}$, caregiver burden, and economic and healthcare burden of $\mathrm{AD}$ by supporting early diagnosis of AD with MTM strategies, early review of emerging therapies, care coordination, and providing covered nonpharmacologic interventions.
Author affiliation: Winston Wong, PharmD, is president of W-Squared Group, Longboat Key, FL.

Funding source: This activity is supported by an educational grant from Biogen MA, Inc.

Author disclosure: Dr Wong has no relevant financial relationships with commercial interests to disclose.

Authorship information: Substantial contributions to the concept and design; drafting of the manuscript; overall supervision; and critical revision of the manuscript for important intellectual content.

Address correspondence to: wwong@wsquaredgroup.com

Medical writing and editorial support: Elenna M. Smalley, PharmD, BCPS

\section{REFERENCES}

1. UCSF Weill Institute for Neurosciences: Memory and Aging Center. What is dementia? Accessed June 11, 2020. memory.ucsf.edu/what-dementia

2. Alzheimer's Association Report. 2020 Alzheimer's disease facts and figures. Alzheimers Dement. 2020;16:391-460. doi: 10.1002/alz.1206

3. Petersen RC. Mild cognitive impairment. Continuum (Minneap Minn). 2016;22(2):404-418. doi: 10.1212/ CON.0000000000000313

4. Albert MS, DeKosky ST, Dickson D, et al. The diagnosis of mild cognitive impairment due to Alzheimer's disease: recommendations from the National Institute on Aging-Alzheimer's Association workgroups on diagnostic guidelines for Alzheimer's disease. Alzheimers Dement. 2011;7(3):270-279. doi: 10.1016/j.jalz.2011.03.008

5. Morris JC, Blennow K, Froelich L, et al. Harmonized diagnostic criteria for Alzheimer's disease: recommendations. J Intern Med. 2014;275(3):204-213. doi: 10.1111/joim.12199

6. Food and Drug Administration; US Department of Health and Human Services. Early Alzheimer's disease: developing drugs for treatment; guidelines for industry. February 2018. Accessed February 20, 2020. fda.gov/media/110903/download

7. NIH: National Institute on Aging. What is mild cognitive impairment? Updated May 17, 2017. Accessed June 11, 2020. nia.nih.gov/health/what-mild-cognitive-impairment

8. Centers for Disease Control and Prevention. What is the burden of Alzheimer's disease in the United States? Updated September 20, 2019. Accessed April 30, 2020. cdc.gov/aging/aginginfo/alzheimers. htm\#burden

9. Prabhune K, Pande A, Mahadik B, Paratane D. Healthcare costs associated with Alzheimer's disease in the US and EU-5-a systematic literature review. 2020-05, ISPOR 2020. Abstract.

10. Cordell CB, Borson S, Boustani M, et al; Medicare Detection of Cognitive Impairment Workgroup. Alzheimer's Association recommendations for operationalizing the detection of cognitive impairment during the Medicare Annual Wellness Visit in a primary care setting. Alzheimers Dement. 2013;9(2):141-150. doi: 10.1016/j.jalz.2012.09.011

11. Galvin JE, Sadowsky CH; NINCDS-ADRDA. Practical guidelines for the recognition and diagnosis of dementia. J Am Board Fam Med. 2012-25(3):367-382. doi: 10.3122/jabfm.2012.03.100181

12. Cure Alzheimer's Fund. Stats \& costs. Accessed April 30, 2020. curealz.org/the-disease/statsand-costs/

13. Deb A, Thornton JD, Sambamoorthi U, Innes K. Direct and indirect cost of managing Alzheimer's disease and related dementias in the United States. Expert Rev Pharmacoecon Outcomes Res. 2017;17(2):189202. doi: 10.1080/14737167.2017.1313118

14. Pyenson B, Sawhney TG, Steffens C, Rotter D, et al. The real-world Medicare costs of Alzheimer disease: considerations for policy and care. J Manag Care Spec Pharm. 2019;25(7):800-809. doi: 10.18553/ jmcp.2019.25.7.800

15. Kelley AS, McGarry K, Gorges, R, Skinner JS. The burden of health care costs for patients with dementia in the last 5 years of life. Ann Intern Med. 2015;163(10):729-736. doi: 10.7326/M15-0381

16. Yang Z, Zhang K, Lin PJ, Clevenger C, Atherly A. A longitudinal analysis of the lifetime cost of dementia. Health Serv Res. 2012;47(4):1660-1678. doi: 10.1111/j.1475-6773.2011.01365x

17. Lin PJ, Zhong Y, Fillit HM, Chen E, Neumann PJ. Medicare expenditures of individuals with Alzheimer's disease and related dementias or mild cognitive impairment before and after diagnosis. J Am Geriatr Soc. 2016;64(8):1549-1557. doi: 10.1111/jgs.14227

18. Goldfeld KS, Stevenson DG, Hamel MB, Mitchell SL. Medicare expenditures among nursing home residents with advanced dementia. Arch Intern Med. 2011;171(9):824-830. doi: 10.1001/archinternmed.2010.478 19. Dwibedi N, Findley PA, Wiener RC, Shen C, Sambamoorthi U. Alzheimer disease and related disorders and out-of-pocket healthcare spending and burden among elderly Medicare beneficiaries. Med Care. 2018;56(3):240-246. doi: 10.1097/MLR.0000000000000869

20. El-Hayek YH, Wiley RE, Khoury CP, et al. Tip of the iceberg: assessing the global socioeconomic costs of Alzheimer's disease and related dementias and strategic implications for stakeholders. J Alzheimers Dis. 2019;70(2):323-341. doi: 10.3233/JAD-190426

21. Zhu CW, Scarmeas N, Torgan R, et al. Home health and informal care utilization and costs over time in Alzheimer's disease. Home Health Care Serv Q. 2008;27(1):1-20. doi: 10.1300/J027v27n01_01

22. Rattinger GB, Schwartz S, Mullins CD, et al. Dementia severity and the longitudinal costs of informal care in the Cache County population. Alzheimers Dement. 2015;11(8):946-954. doi: 10.1016/j.jalz.2014.11.004

23. Coe NB, Skira MM, Larson EB. A comprehensive measure of the costs of caring for a parent: differences according to functional status. Am Geriatr Soc. 2018;66(10):2003-2008. doi: 10.1111/jgs.15552 24. Zhu CW, Scarmeas N, Ornstein K, et al. Health-care use and cost in dementia caregivers: longitudinal results from the Predictors Caregiver Study. Alzheimers Dement. 2015;11(4):444-454. doi: 10.1016/j.jalz.2013.12.018 


\section{ECONOMIC BURDEN OF ALZHEIMER DISEASE AND MANAGED CARE CONSIDERATIONS}

25. Seuhs BT, Shah SN, Davis CD, et al. Household members of persons with Alzheimer's disease: health conditions, healthcare resource use, and healthcare costs. J Am Geriatr Soc. 2014;62(3):435-441. doi: 10.1111/jgs.12694

26. Lawton, MP. Quality of life in Alzheimer disease. Alzheimer Dis Assoc Disord. 1994:8(suppl 3):138-150. 27. Mokdad AH, Ballestros K, Echko M, et al; US Burden of Disease Collaborators. The state of US health, 1990-2016: burden of diseases, injuries, and risk factors among US states. JAMA. 2018:319(14):14441472. doi: $10.1001 /$ jama.2018.0158

28. Alzheimer's Association. Health systems and clinicians: Management. Accessed June 11, 2020. alz. org/professionals/health-systems-clinicians/management

29. US Department of Health and Human Services. National Alzheimer's Project Act. Updated July 1. 2020. Accessed July 20, 2020. aspe.hhs.gov/national-alzheimers-project-act

30. Alzheimer's Association International Conference 2018. Press release. First practice guidelines for clinical evaluation of Alzheimer's disease and other dementias for primary and specialty care. July 22, 2018. Accessed May 6, 2020. alz.org/aaic/releases_2018/AAIC18-Sun-clinical-practiceguidelines.asp

31. Alzheimer's Association. Dementia-related behaviors. 2015. Accessed July 20, 2020. alz.org/media/ Documents/dementia-related-behaviors-statement.pdf

32. Alzheimer's Association. Treatments: Medications for memory. Accessed June 11, 2020. alz.org/ alzheimers-dementia/treatments/medications-for-memory

33. Asanad S, Fantini M, Sultan W, et al. Retinal nerve fiber layer thickness predicts CSF amyloid/tau before cognitive decline. PLoS One. 2020;15(5):e0232785. doi: 10.1371/journal.pone.0232785

34. Cordell CB, Borson S, Boustani M, et al; Medicare Detection of Cognitive Impairment Workgroup. Alzheimer's Association recommendations for operationalizing the detection of cognitive impairment during the Medicare Annual Wellness Visit in a primary care setting. Alzheimers Dement. 2013;9(2):141-150. doi: 10.1016/j.jalz.2012.09.011

35. Breitner JC. Mild cognitive impairment and progression to dementia: new findings. Neurology. 2014;82(4):e34-5. doi: 10.1212/WNL.0000000000000158

36. Rassmussen J, Langerman H. Alzheimer's disease-why we need early diagnosis. Degener Neurol Neuromuscul Dis. 2019:9:123-130. doi: 10.2147/DNND.S228939

37. Dubois B, Padovani A, Scheltens P, Rossi A, Dell'Agnello G. Timely diagnosis for Alzheimer's disease: a literature review on benefits and challenges. J Alzheimers Dis. 2016:49(3):617-631. doi: $10.3233 / J A D-150692$

38. Weimer DL, Sager MA. Early identification and treatment of Alzheimer's disease: social and fiscal outcomes. Alzheimers Dement. 2009:5(3):215-226. doi: 10.1016/j.jalz.2009.01.028

39. De Vugt ME, Verhey FR. The impact of early dementia diagnosis and intervention on informal caregivers. Prog Neurobiol. 2013;110:54-62. doi: 10.1016/j.pneurobio.2013.04.005
40. Jun H, Cho SK, Mattke S, Suen SC. How much value should a preventive treatment for Alzheimer's disease offer? Cost-effectiveness thresholds for pricing a disease-modifying therapy. 2020-05, ISPOR 2020. Abstract. Accessed July 20, 2020. ispor.org/heor-resources/presentations-database/presentation/ intl2020-3182/100323

41. Alzheimer's Association. Medicare coverage of care planning for people with cognitive impairment. Updated February 2020. Accessed May 5, 2020. alz.org/media/Documents/alzheimers-dementia-medicare-coverage-for-careplanners-ts.pdf

42. American Pharmacists Association. Medication therapy management (MTM) services. Accessed May 9. 2020. pharmacist.com/medication-therapy-management-services

43. Allen K. BrightFocus Foundation. Caregiver training: what you need to know. Accessed June 11, 2020. brightfocus.org/alzheimers/article/caregiver-training-what-you-need-know

44. Millican HH, Piper BJ. Medicare Advantage risk score primer: what you need to know about diagnoses supporting risk scores and revenue payment timing. Milliman White Paper. October 9, 2017. Accessed July 20, 2020. us.milliman.com/en/insight/medicare-advantage-risk-score-primer-what-you-need-toknow-about-diagnoses-supporting-ris

45. Pyenson BS, Steffens C. Including dementia in the Part C Medicare risk adjuster: health services issues. Milliman White Paper. February 2019. Accessed July 20, 2020. milliman.com/-/media/Milliman/ importedfiles/uploadedFiles/insight/2019/including-dementia-medicare-risk-adjuster.ashx

46. Petersen RC, Lopez O, Armstrong MJ, et al. Practice guideline update summary: mild cognitive impairment: Report of the Guideline Development, Dissemination, and Implementation Subcommittee of the American Academy of Neurology. Neurology. 2018:90(3):126-135. doi: 10.1212/WNL.0000000000004826 47. American Physical Therapy Association. ChoosePT Guide: Physical therapy guide to Alzheimer's disease. Accessed June 11, 2020. choosept.com/symptomsconditionsdetail/physical-therapy-guide-toalzheimers-disease

48. American Occupational Therapy Association. Alzheimer's disease tip sheet. Accessed June 11, 2020. aota.org/About-Occupational-Therapy/Patients-Clients/Adults/Alzheimers.aspx

49. RenewActive by UnitedHealthcare. Updated May 27, 2020. Accessed June 11, 2020. uhcrenewactive. $\mathrm{com} / \mathrm{brain}$

50. BrainHQ brain exercises spread across 2019 Medicare Advantage plans. October 15, 2018. Accessed July 20, 2020. brainhq.com/news/brainhq-brain-exercises-spread-across-2019-medicare-advantage-plans 51. Spector A, Orrell M. Woods B. Cognitive stimulation therapy (CST): effects on different areas of cognitive function for people with dementia. Int J Geriatr Psychiatry. 2010;25(12):1253-1258. doi: 10.1002/gps.2464 52. Prizer LP, Zimmerman S. Progressive support for activities of daily living for persons living with dementia. Gerontologist. 2018;58(suppl 1):S74-S87. doi: 10.1093/geront/gnx103

53. North Coast Medical \& Rehabilitation Products. Occupational therapy: assistive devices (ADL). Accessed July 20, 2020. ncmedical.com/categories/Assistive-Devices-ADL_12839533.html 


\section{Addressing Unmet Needs in Alzheimer Disease: Implications of Delayed Diagnosis and Examining New and Emerging Therapies}

Release date: August 7, 2020

Expiration date: August 7, 2021

Pharmacy Credit

Instructions for Receiving Continuing Pharmacy Education (CPE) Credit: Testing Information

This lesson is free online; request your CE credit at www.PharmacyTimes.org/go/early-AD-suppl.

Sample of Online Posttest

Choose the best answer for each of the following:

1. Which of the following are currently available biomarkers used in the diagnosis and monitoring of the Alzheimer disease (AD) continuum?

A. A $\beta$ positron emission tomography (PET) scanning, neurodegeneration on magnetic resonance imaging (MRI), and the Mini-Mental Status Exam (MMSE)

B. $A \beta$ PET scanning, $A \beta$ cerebrospinal fluid testing, and neurodegeneration on MRI

C. A $\beta$ PET scanning, neurodegeneration on MRI, and apolipoprotein E (APOE) genotyping

D. A $\beta$ PET scanning, APOE genotyping, and the MMSE

2. In observational studies, up to what percentage of patients with mild cognitive impairment progressed to dementia over 5 years of follow-up?
A. $21 \%$
B. $26 \%$
C. $38 \%$
D. $57 \%$

3. Which of the following statements most accurately reflects the effects of cholinesterase inhibitors and memantine on the course of the AD continuum?

A. Although cholinesterase inhibitors do not change the course of $\mathrm{AD}$, memantine slows the progression of dementia.

B. Cholinesterase inhibitors slow the course of AD, but memantine does not.

C. Cholinesterase inhibitors and memantine do not slow the progression of $\mathrm{AD}$.

D. Cholinesterase inhibitors and memantine slow the course of AD.

\section{Testing Directions}

1. Each participant evaluating the activity is eligible to receive CE credit

2. To receive your credit online, go to www.PharmacyTimes.org/go/ early-AD-suppl and complete the online posttest and the online activity evaluation form before the expiration date. Your CE credit will be automatically uploaded to CPE Monitor. Please ensure that your Pharmacy Times $^{\circledast}$ account is updated with your NABP e-profile ID number and your date of birth (MMDD format). Participation data will not be uploaded into CPE Monitor if you do not have your NABP e-profile ID number and date of birth entered into your profile on www.PharmacyTimes.org.

4. Which phase 3 investigational drug for $A D$ targets inflammation?
A. ALZT-OP1
B. AGB101
C. $\mathrm{CAD} 106$
D. BAN2401

5. Which phase 3 investigational drug for $A D$ is a monoclonal antibody that targets aggregated $A \beta$ fibrils and soluble oligomers?
A. LMTX
B. COR388
C. CAD106
D. Aducanumab

6. Which statement is true?
A. Early diagnosis of AD is associated with improved quality of life.
B. It is estimated that informal caregiver costs increase $5 \%$ per year as symptoms of AD progress.
C. Early intervention is not associated with improvements in cognitive function.
D. Long-term care is not a major driver of cost of care in AD.

7. Which of the following is true about the economic impact of direct costs of care for patients with AD?
A. As the prevalence of AD increases, costs of care are estimated to decrease.
B. Total costs of care for patients with $\mathrm{AD}$ are estimated to increase to more than $\$ 1$ trillion by 2050 .
C. Total Medicare payments in 2019 for patients with AD were similar to those without AD.
D. Patient out-of-pocket costs were similar in patients with $\mathrm{AD}$ compared with those without AD. 


\section{Informal caregiving for patients with $A D$}

A. Is associated with poor economic outcomes for the caregiver.

B. Is similar to informal caregiving for patients with other diseases.

C. Is not associated with increased healthcare utilization by the caregiver.

D. Does not pose a significant financial burden on the caregiver.
9. Which of the following has been shown to reduce healthcare costs and improve quality of life of patients with $A D$ and their caregivers?
A. Speech therapy
B. Medication therapy management
C. Physical therapy
D. Assistive devices for activities of daily living

10. Outcomes associated with early diagnosis of $A D$ include all of the following, except:
A. Increased costs
B. Early pharmacologic and nonpharmacologic interventions
C. Increased patient and caregiver quality of life
D. Inclusion of the patient in the planning of future care needs 


\title{
SUPPLEMENT POLICY STATEMENT
}

\author{
Standards for Supplements to The American Journal of Managed Care ${ }^{\circledR}$
}

All supplements to The American Journal of Managed Care ${ }^{\circledR}$ are designed to facilitate and enhance ongoing medical education in various therapeutic disciplines. All Journal supplements adhere to standards of fairness and objectivity, as outlined below. Supplements to The American Journal of Managed Care ${ }^{\circledR}$ will:

I. Be reviewed by at least 1 independent expert from a recognized academic medical institution.

II. Disclose the source of funding in at least 1 prominent place.

III. Disclose any existence of financial interests of supplement contributors to the funding organization.

IV. Use generic drug names only, except as needed to differentiate between therapies of similar class and indication.

V. Be up-to-date, reflecting the current las of date of publication) standard of care.

VI. Be visually distinct from The American Journal of Managed Care ${ }^{\circledR}$.

VII. Publish information that is substantially different in form and content from that of the accompanying edition of The American Journal of Managed Care ${ }^{\circledR}$.

VIII. Prohibit excessive remuneration for contributors and reviewers.

IX. Carry no advertising.

Publisher's Note: The opinions expressed in this supplement are those of the authors, presenters, and/or panelists and are not attributable to the sponsor or the publisher, editor, or editorial board of The American Journal of Managed Care ${ }^{\circledR}$. Clinical judgment must guide each professional in weighing the benefits of treatment against the risk of toxicity. Dosages, indications, and methods of use for products referred to in this supplement are not necessarily the same as indicated in the package insert for the product and may reflect the clinical experience of the authors, presenters, and/or panelists or may be derived from the professional literature or other clinical sources. Consult complete prescribing information before administering. 
